\title{
Dimeric Diarylheptanoids with Neuroprotective Activities from Rhizomes of Alpinia officinarum
}

Hui Liu 1,2,3,\#, Xiaojun Wang,2,3,\#, Qiaoyun Shi²,3,\#, Liuren Li ${ }^{2,3}$, Qinghua Zhang ${ }^{2,3}$, Zhen-Long Wu',2,3, Xiao-Jun Huang ${ }^{1,2,3}$, Qing-Wen Zhang ${ }^{4}$, Wen-Cai Ye ${ }^{1,2,3}$, Ying Wang ${ }^{1,2,3, *}$, and Lei Shi ${ }^{2,3, *}$

${ }^{1}$ Institute of Traditional Chinese Medicine \& Natural Products, Jinan University, Guangzhou 510632, People's Republic of China

2 JNU-HKUST Joint Laboratory for Neuroscience \& Innovative Drug Research, Jinan University, Guangzhou 510632, People's Republic of China

${ }^{3}$ Guangdong Province Key Laboratory of Pharmacodynamic Constituents of TCM \& New Drugs Research, Jinan University, Guangzhou 510632, People’s Republic of China

${ }^{4}$ State Key Laboratory of Quality Research in Chinese Medicine, Institute of Chinese Medical Sciences, University of Macau, Macao, People's Republic of China

\# These authors contributed equally to this study: Hui Liu, Xiaojun Wang, Qiaoyun Shi

Corresponding authors:

Ying Wang, PhD, Professor (wangying_cpu@163.com)

Lei Shi, PhD, Professor (sophielshi80@gmail.com or t shilei@jnu.edu.cn) 


\section{Supporting Information}

\section{Table of Contents}

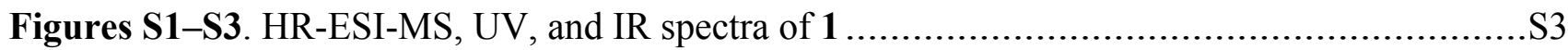

Figures S4-S10. 1D and 2D NMR spectra of 1 ................................................................................ 4

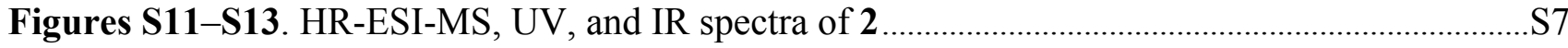

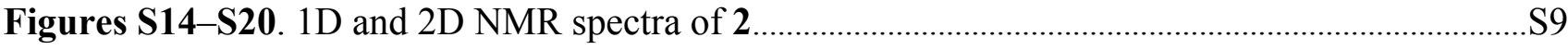

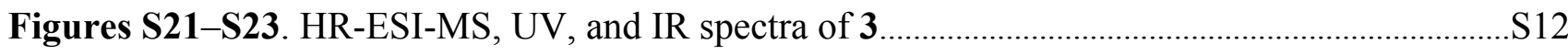

Figures S24-S30. 1D and 2D NMR spectra of 3 ..........................................................................

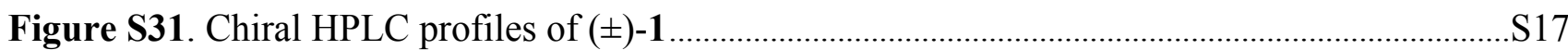

Table S1. DP4+ probability analyses of calculated and experimental ${ }^{1} \mathrm{H}$ NMR data of $\mathbf{1}$.............S18

Table S2. DP4+ probability analyses of calculated and experimental ${ }^{13} \mathrm{C}$ NMR data of 1 ..................S20

Figures S32-S33. Key molecular orbitals involved in important transitions regarding the ECD spectra of dominant conformers of $\mathbf{1}$ and $\mathbf{2}$ at the B3LYP/6-31+G(d) level in the gas phase ...........S21

Tables S3-S4. Key transitions and their related rotatory and oscillator strengths of dominant conformers of $\mathbf{1}$ and $\mathbf{2}$ at the B3LYP/6-31+G(d) level in the gas phase S22

Tables S5-S6. Cartesian coordinate of the dominant conformers of all plausible stereoisomers of $\mathbf{1}$ and 2 


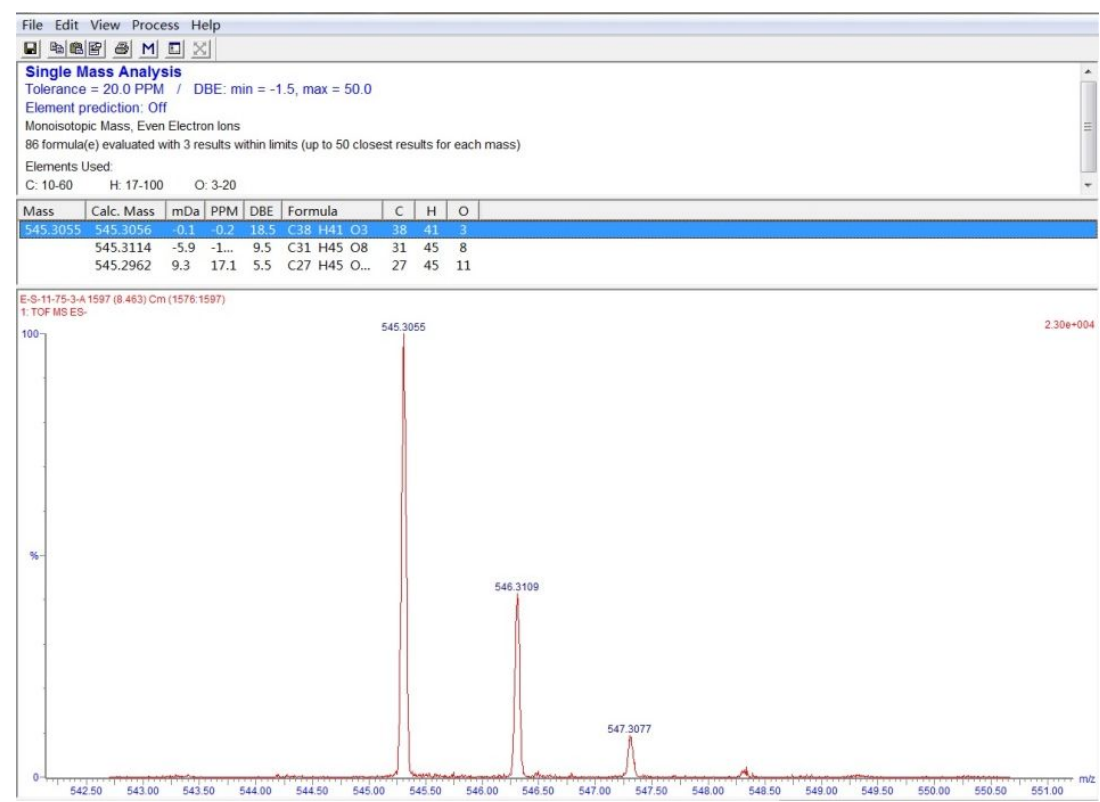

Figure S1. HR-ESI-MS spectrum of 1.

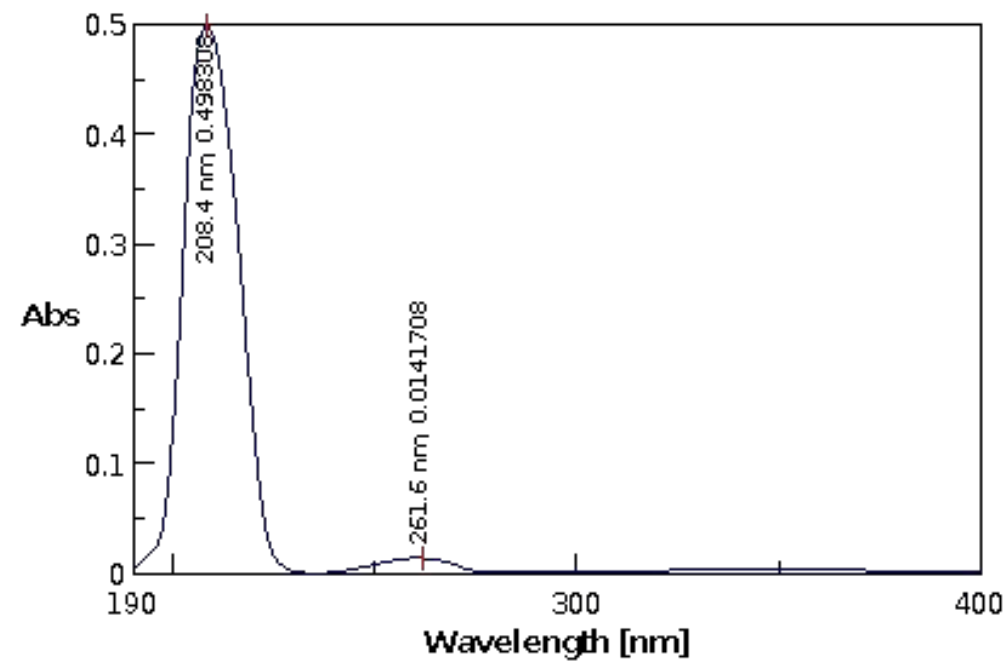

Figure S2. UV spectrum (MeOH) of $\mathbf{1}$.

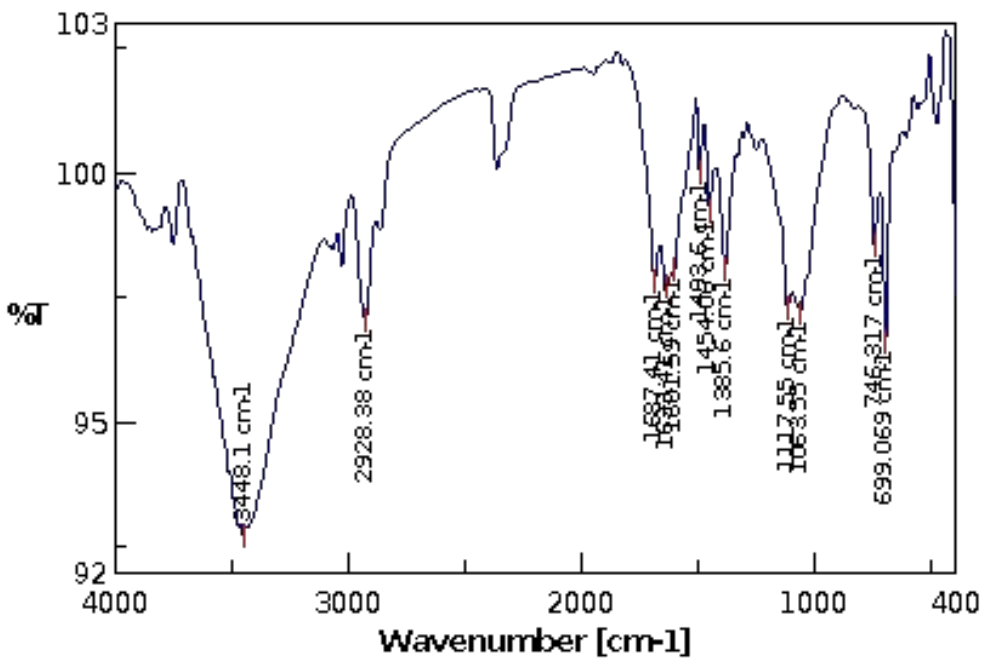

Figure S3. IR spectrum (KBr) of 1 . 


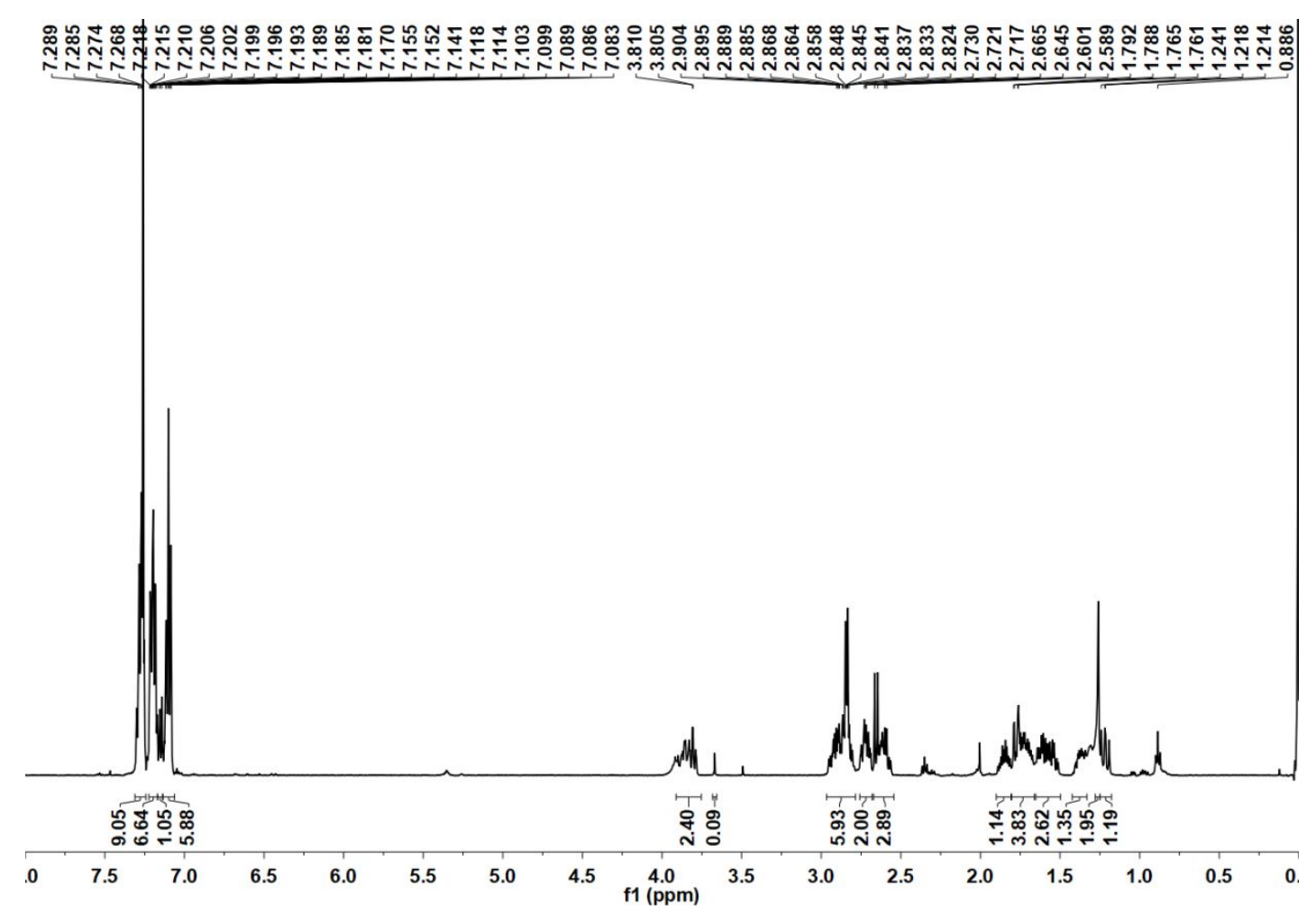

Figure S4. ${ }^{1} \mathrm{H}$ NMR spectrum of 1 in $\mathrm{CDCl}_{3}(500 \mathrm{MHz})$.

্ֻল్

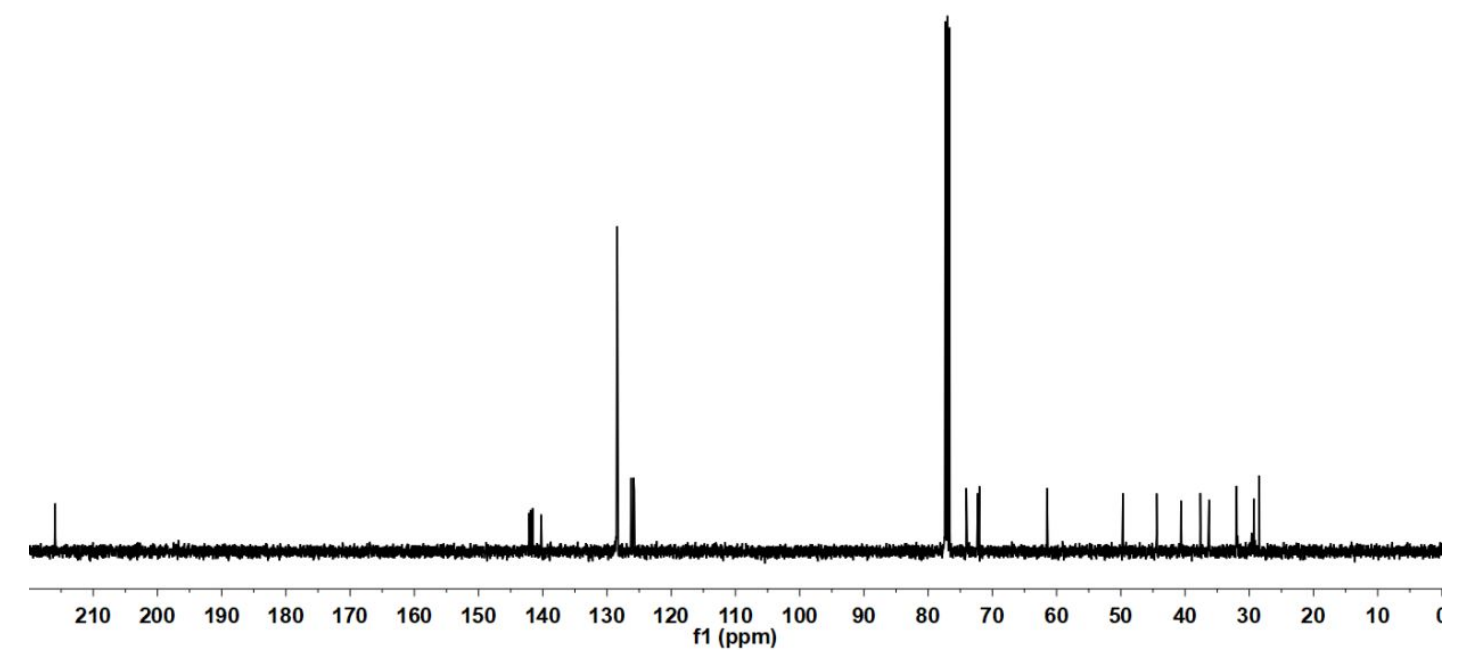

Figure S5. ${ }^{13} \mathrm{C}$ NMR spectrum of 1 in $\mathrm{CDCl}_{3}(125 \mathrm{MHz})$. 


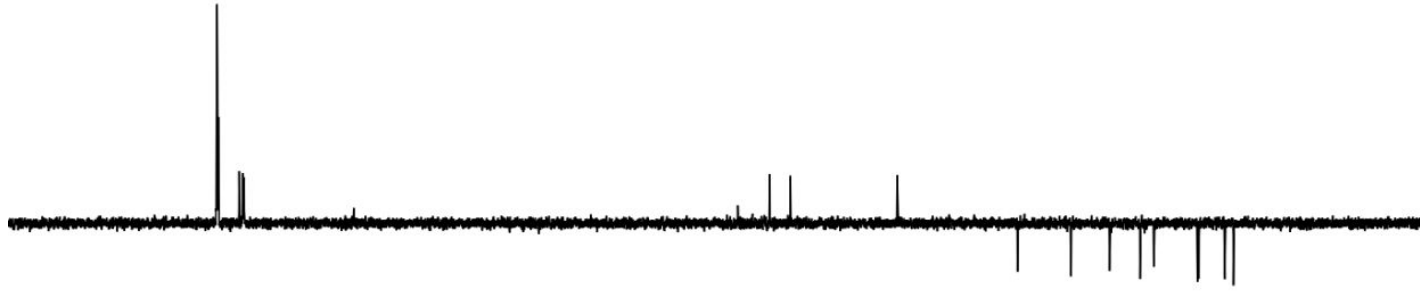

Figure S6. DEPT-135 spectrum of 1 in $\mathrm{CDCl}_{3}(125 \mathrm{MHz})$.

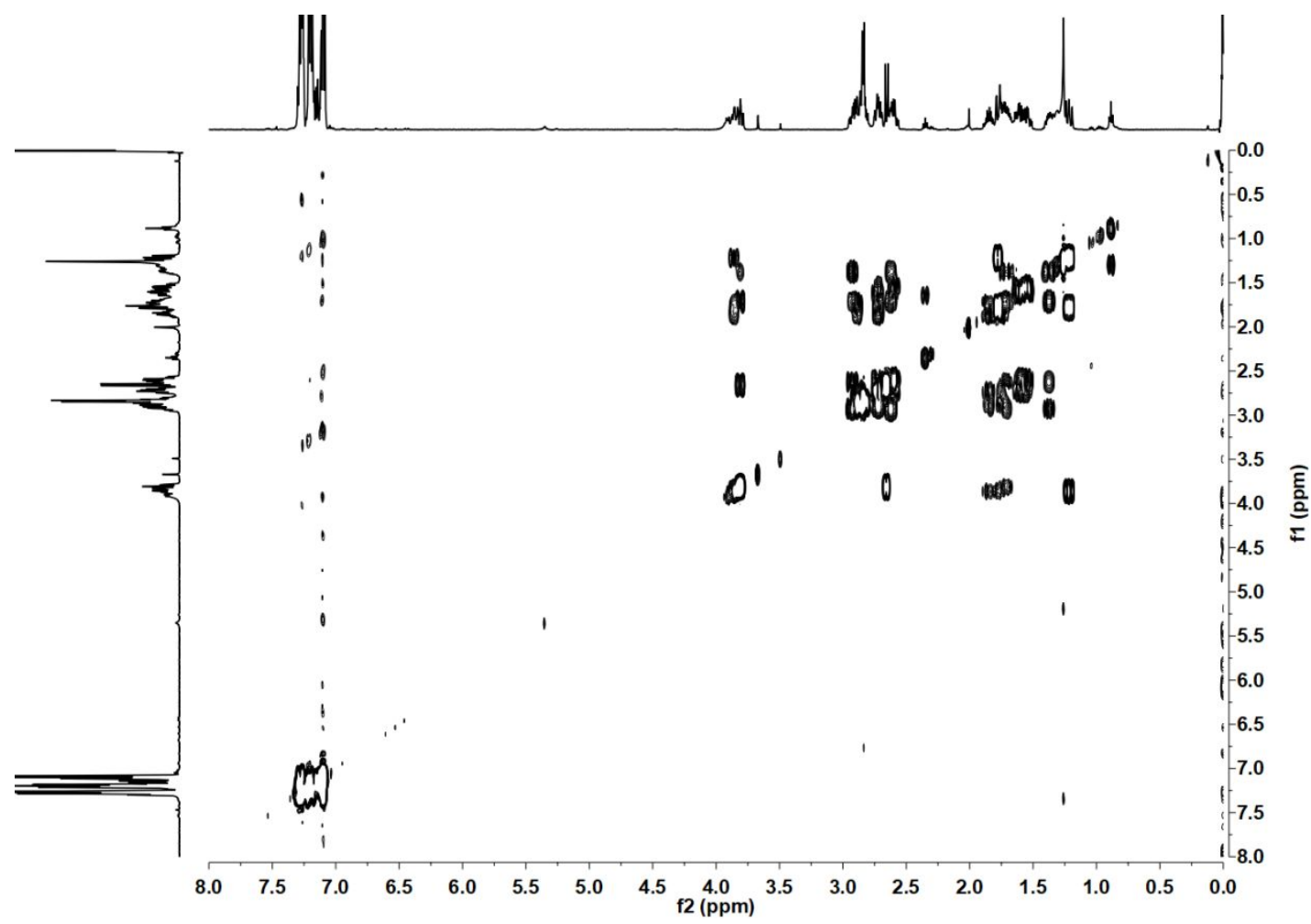

Figure S7. ${ }^{1} \mathrm{H}-{ }^{1} \mathrm{H}$ COSY spectrum of $\mathbf{1}$ in $\mathrm{CDCl}_{3}$. 


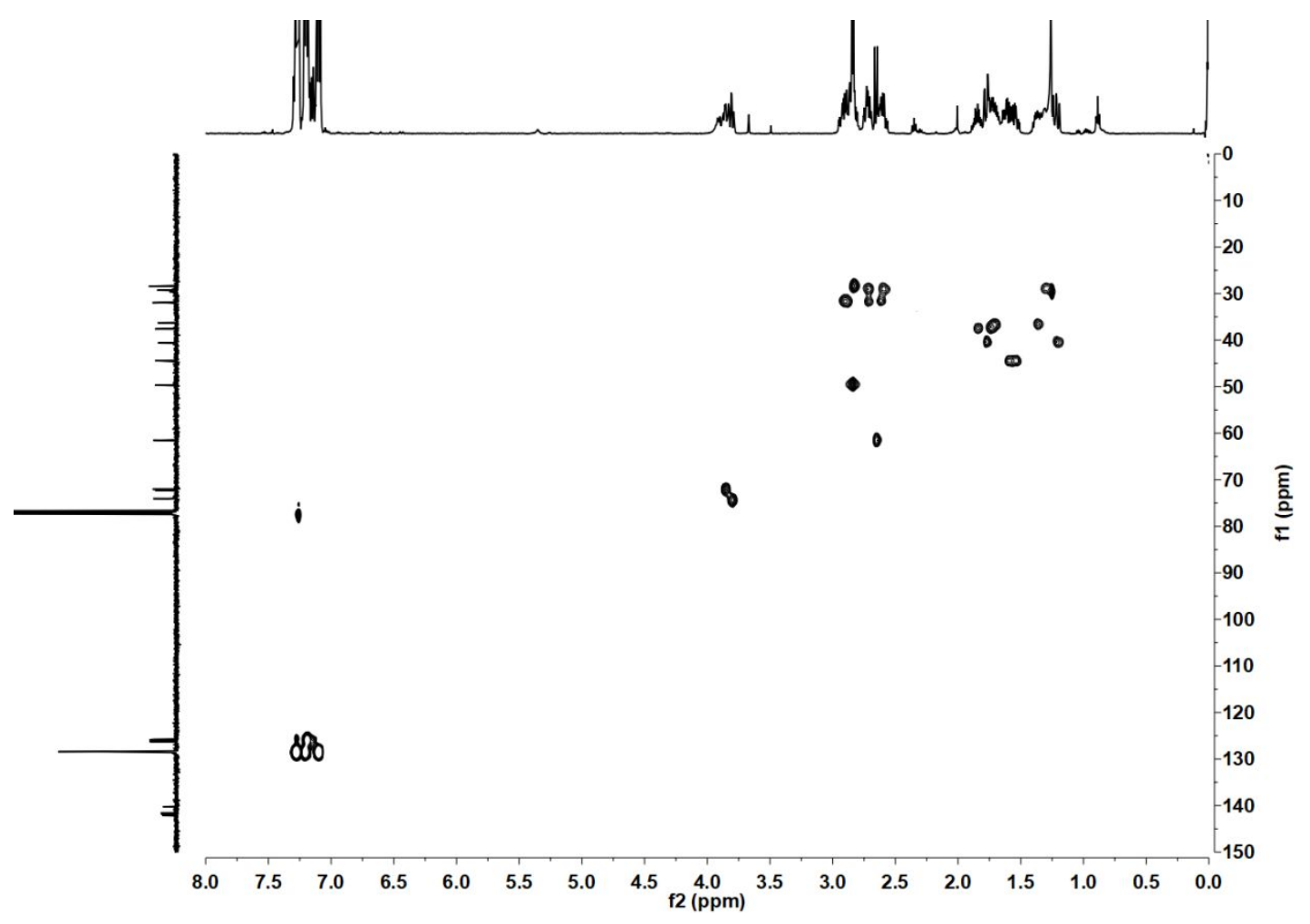

Figure S8. HSQC spectrum of $\mathbf{1}$ in $\mathrm{CDCl}_{3}$.

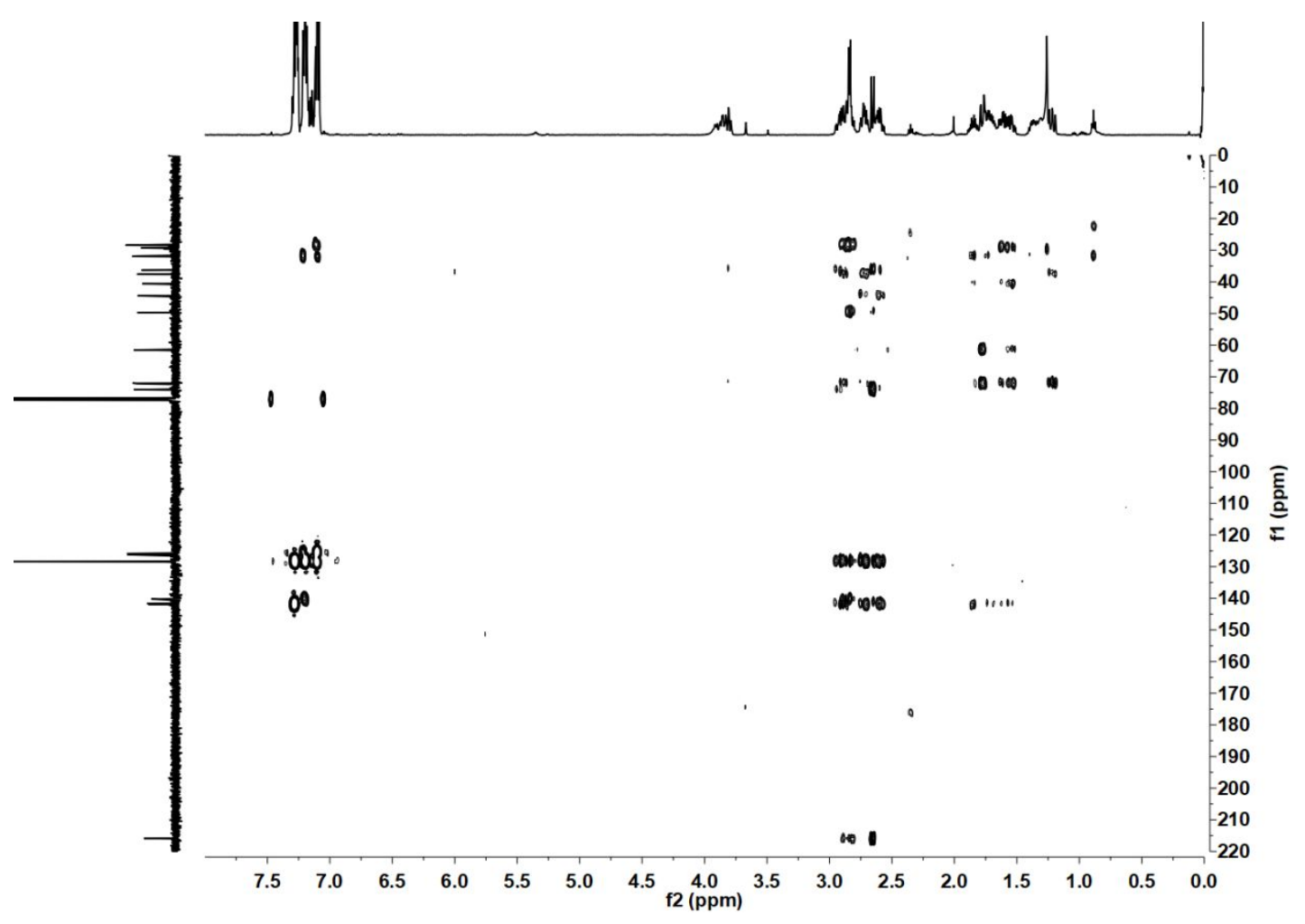

Figure S9. $\mathrm{HMBC}$ spectrum of $\mathbf{1}$ in $\mathrm{CDCl}_{3}$. 


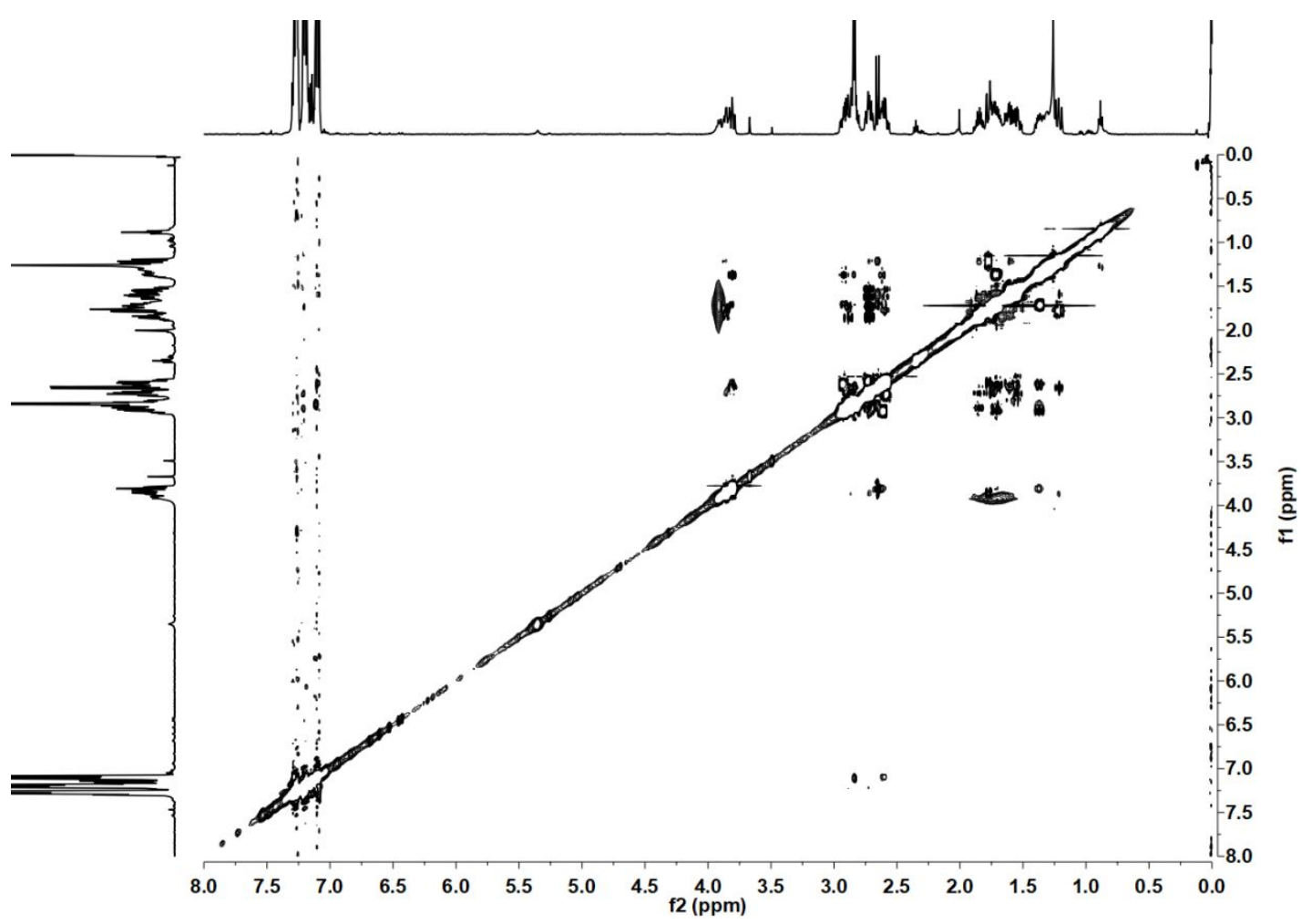

Figure S10. NOESY spectrum of 1 in $\mathrm{CDCl}_{3}$.

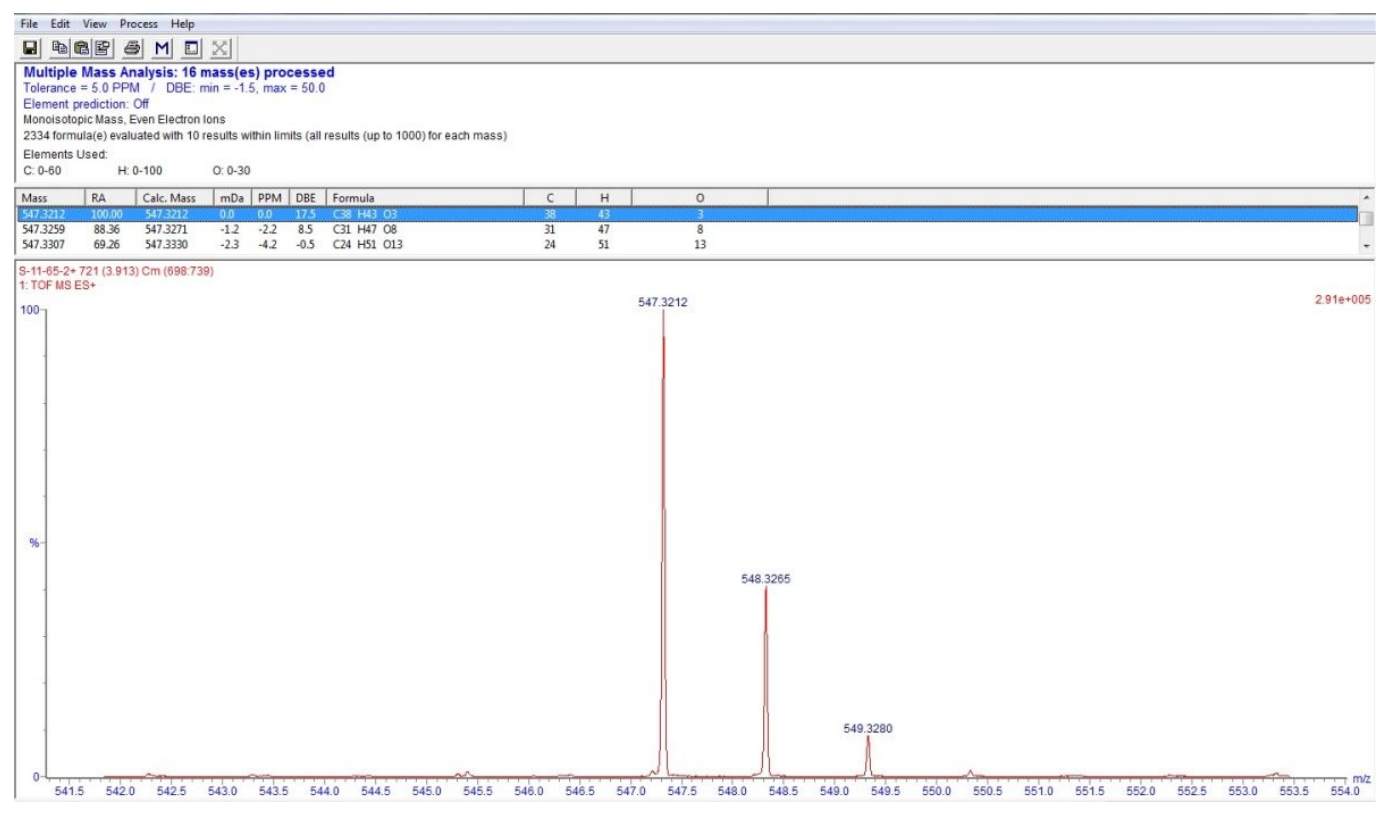

Figure S11. HR-ESI-MS spectrum of 2. 


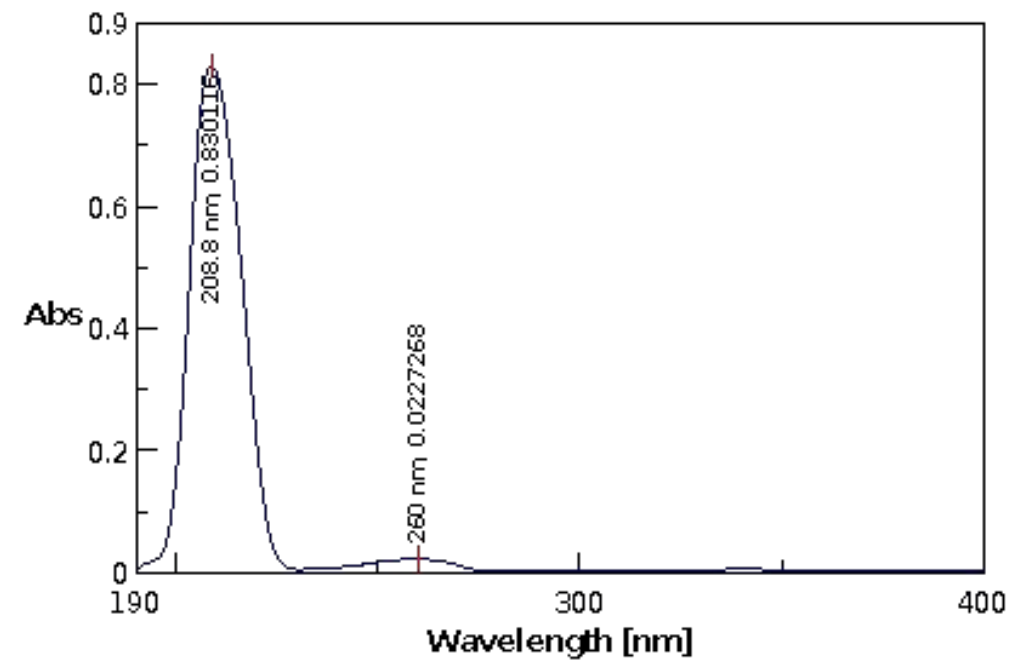

Figure S12. UV spectrum (MeOH) of 2.

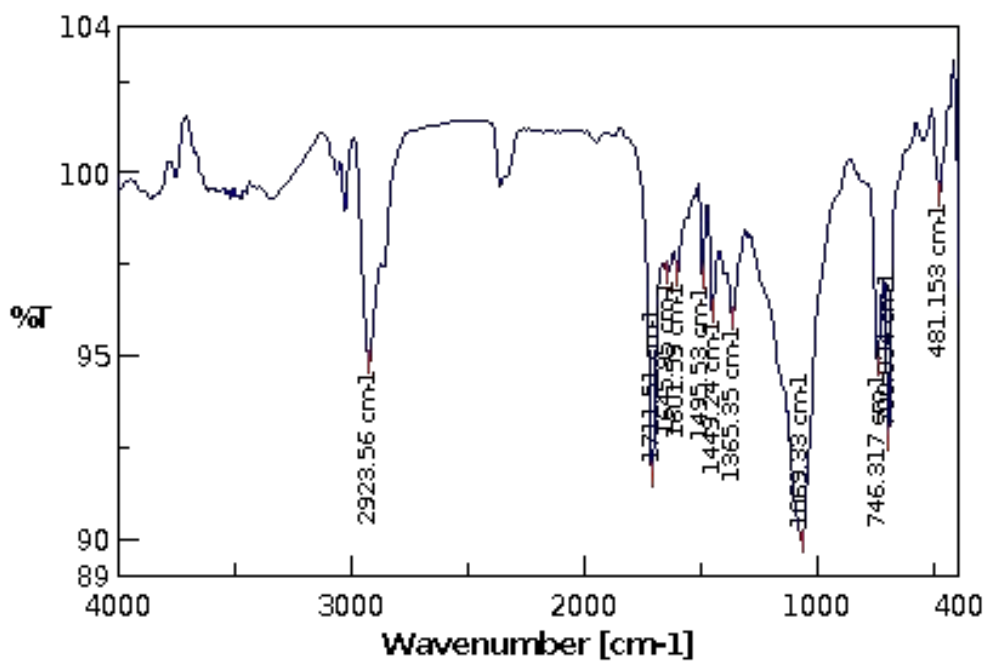

Figure S13. IR spectrum (KBr) of 2. 


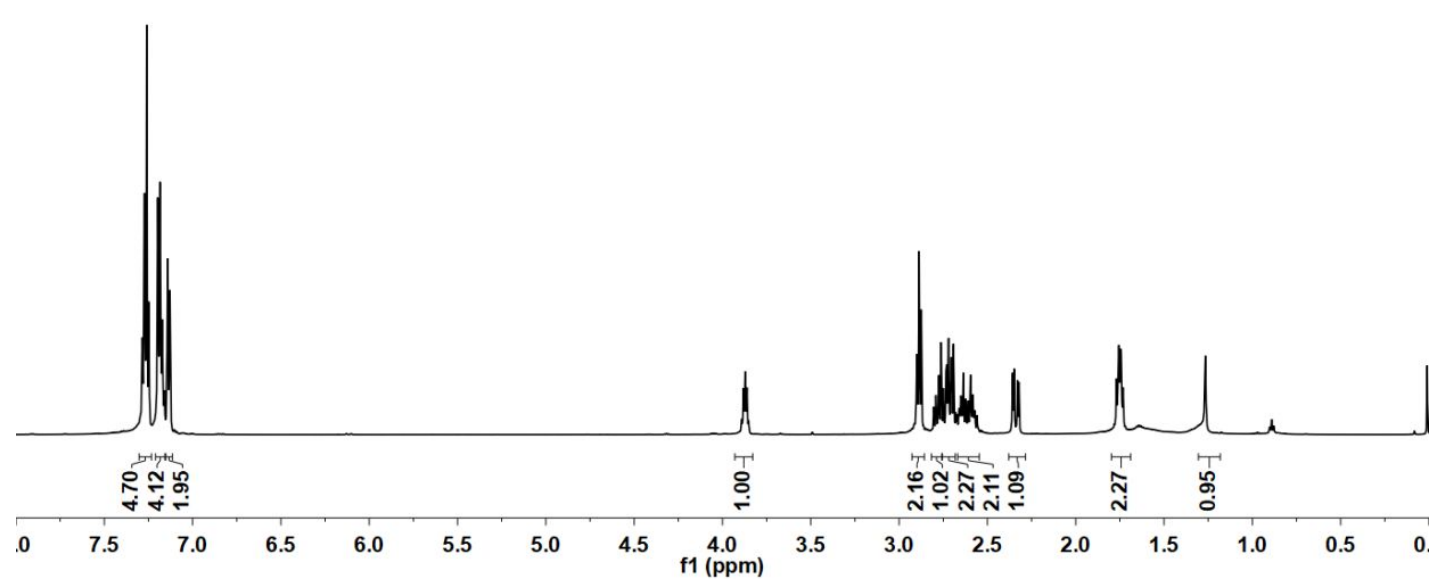

Figure S14. ${ }^{1} \mathrm{H}$ NMR spectrum of 2 in $\mathrm{CDCl}_{3}(600 \mathrm{MHz})$.
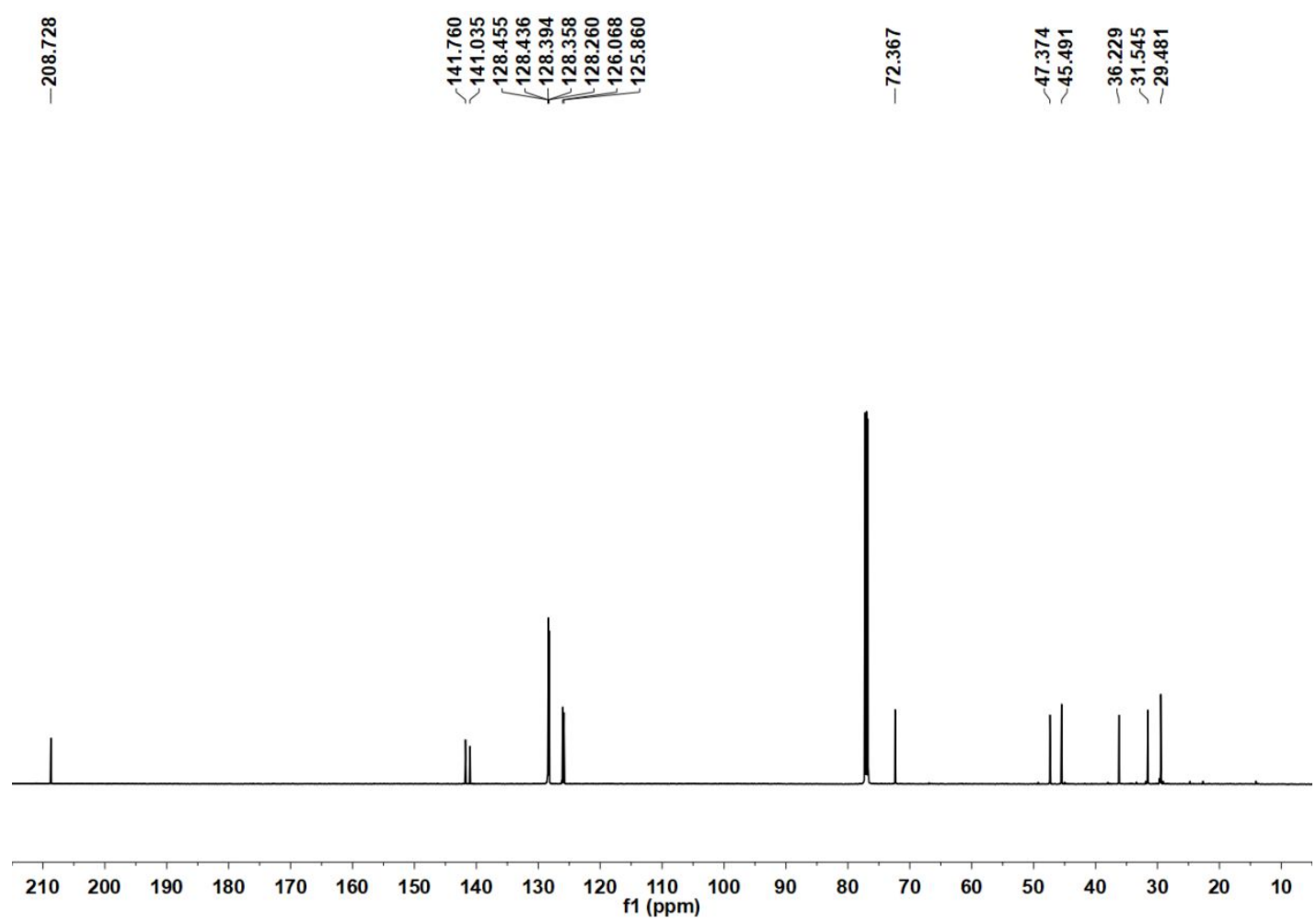

Figure S15. ${ }^{13} \mathrm{C}$ NMR spectrum of 2 in $\mathrm{CDCl}_{3}(150 \mathrm{MHz})$. 


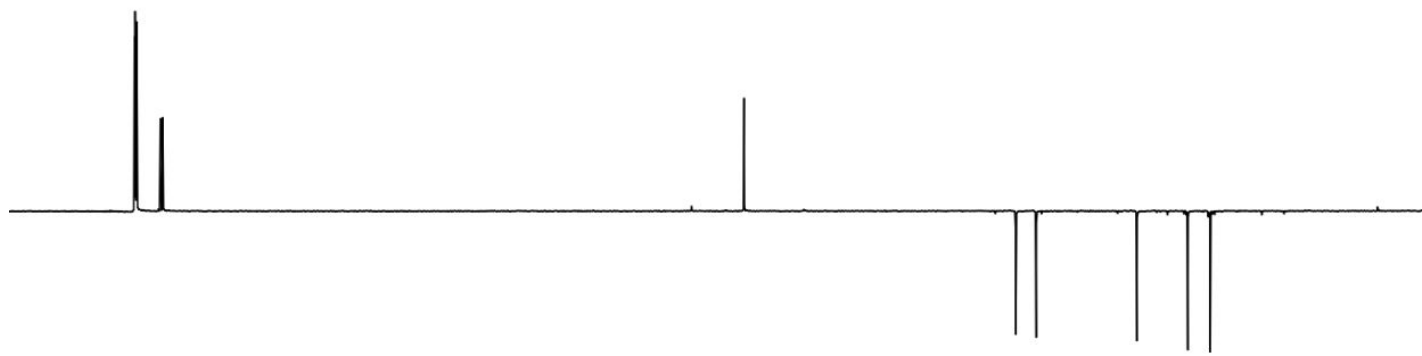

$\begin{array}{lllllllllllllllllllllllllllllll}10 & 135 & 130 & 125 & 120 & 115 & 110 & 105 & 100 & 95 & 90 & 85 & 80 & \begin{array}{c}75 \\ \mathrm{f} 1(\mathrm{ppm})\end{array} & 70 & 65 & 60 & 55 & 50 & 45 & 40 & 35 & 30 & 25 & 20 & 15 & 1\end{array}$

Figure S16. DEPT-135 spectrum of 2 in $\mathrm{CDCl}_{3}(150 \mathrm{MHz})$.

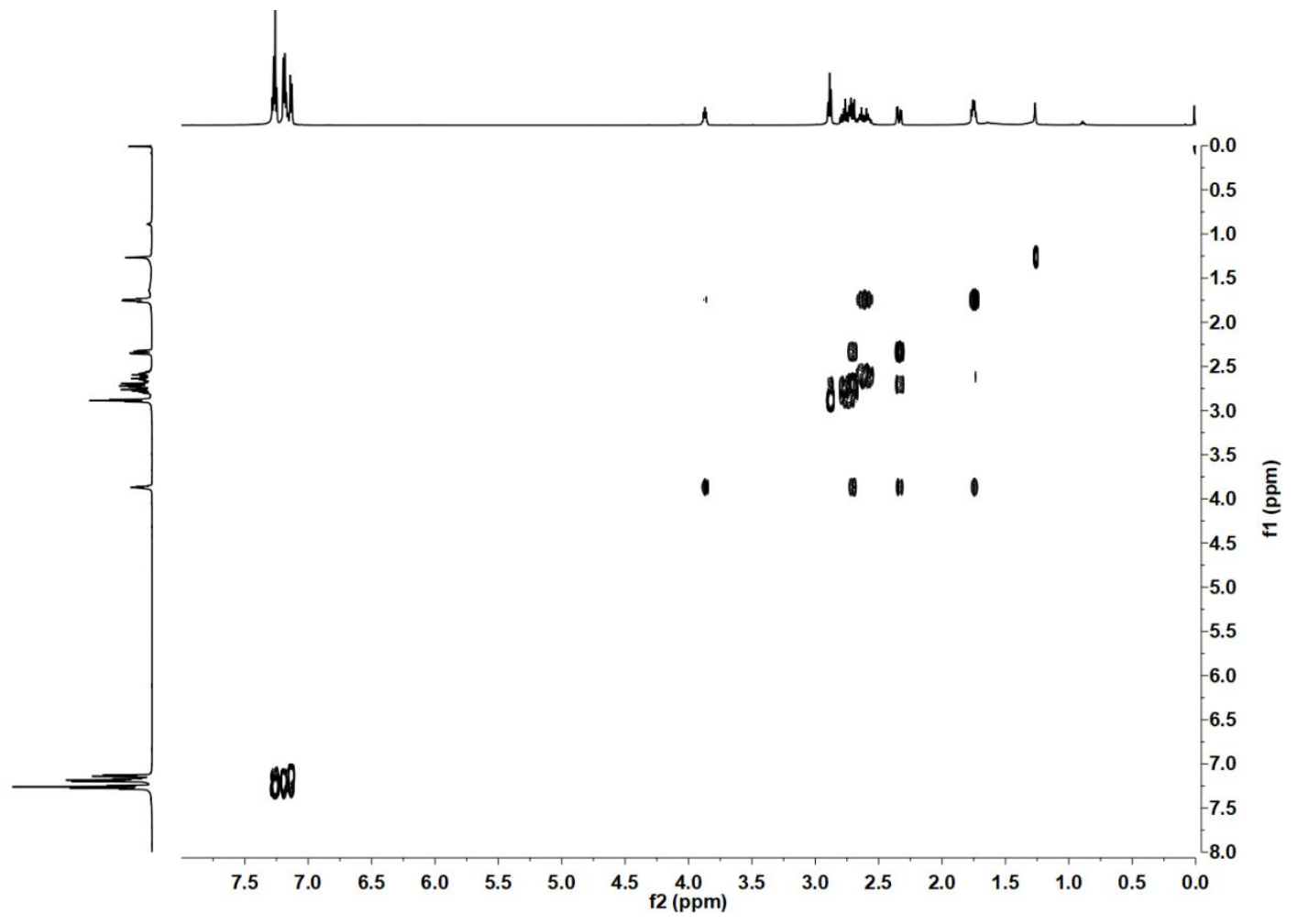

Figure S17. ${ }^{1} \mathrm{H}-{ }^{1} \mathrm{H}$ COSY spectrum of 2 in $\mathrm{CDCl}_{3}$. 


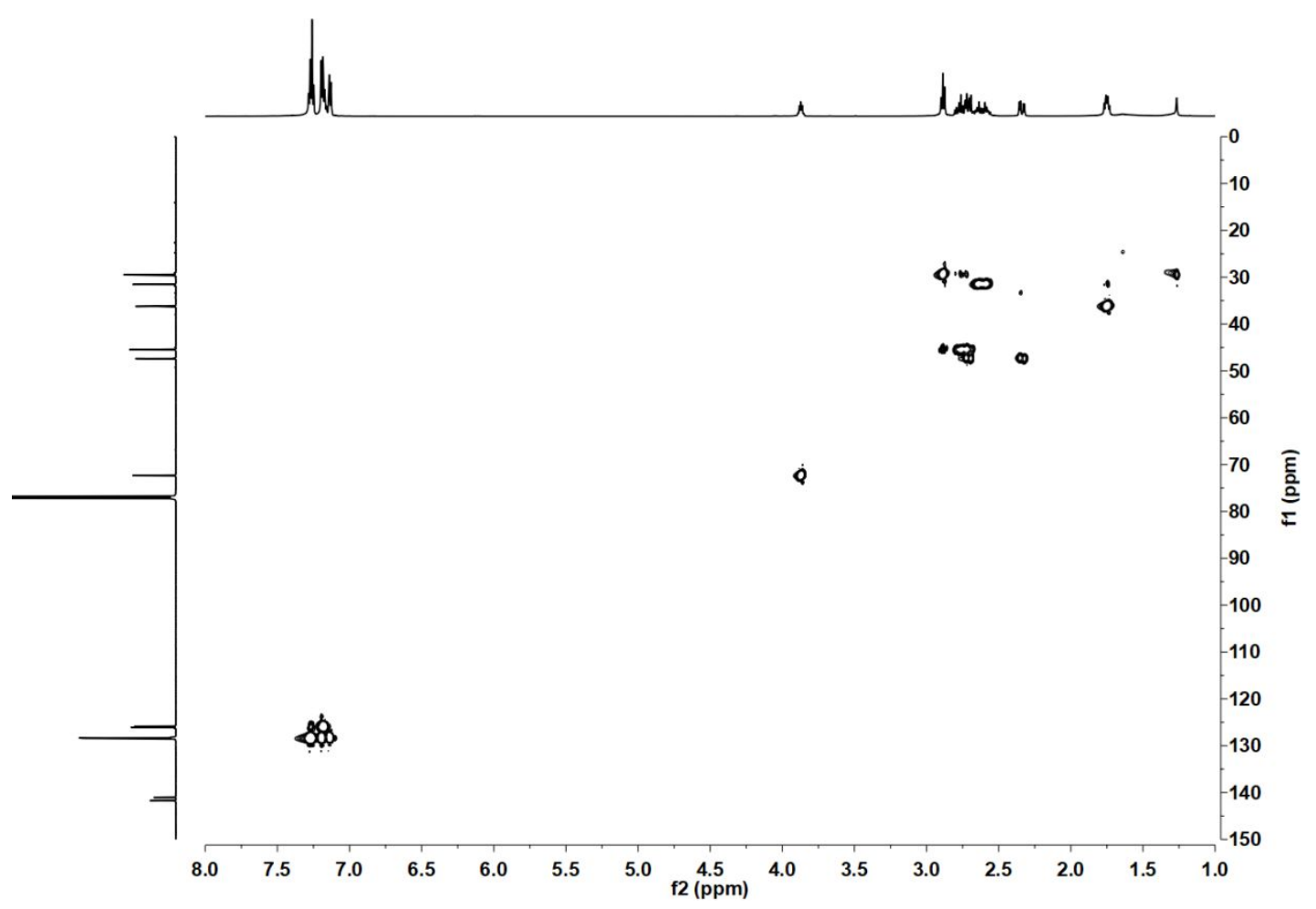

Figure S18. HSQC spectrum of 2 in $\mathrm{CDCl}_{3}$.

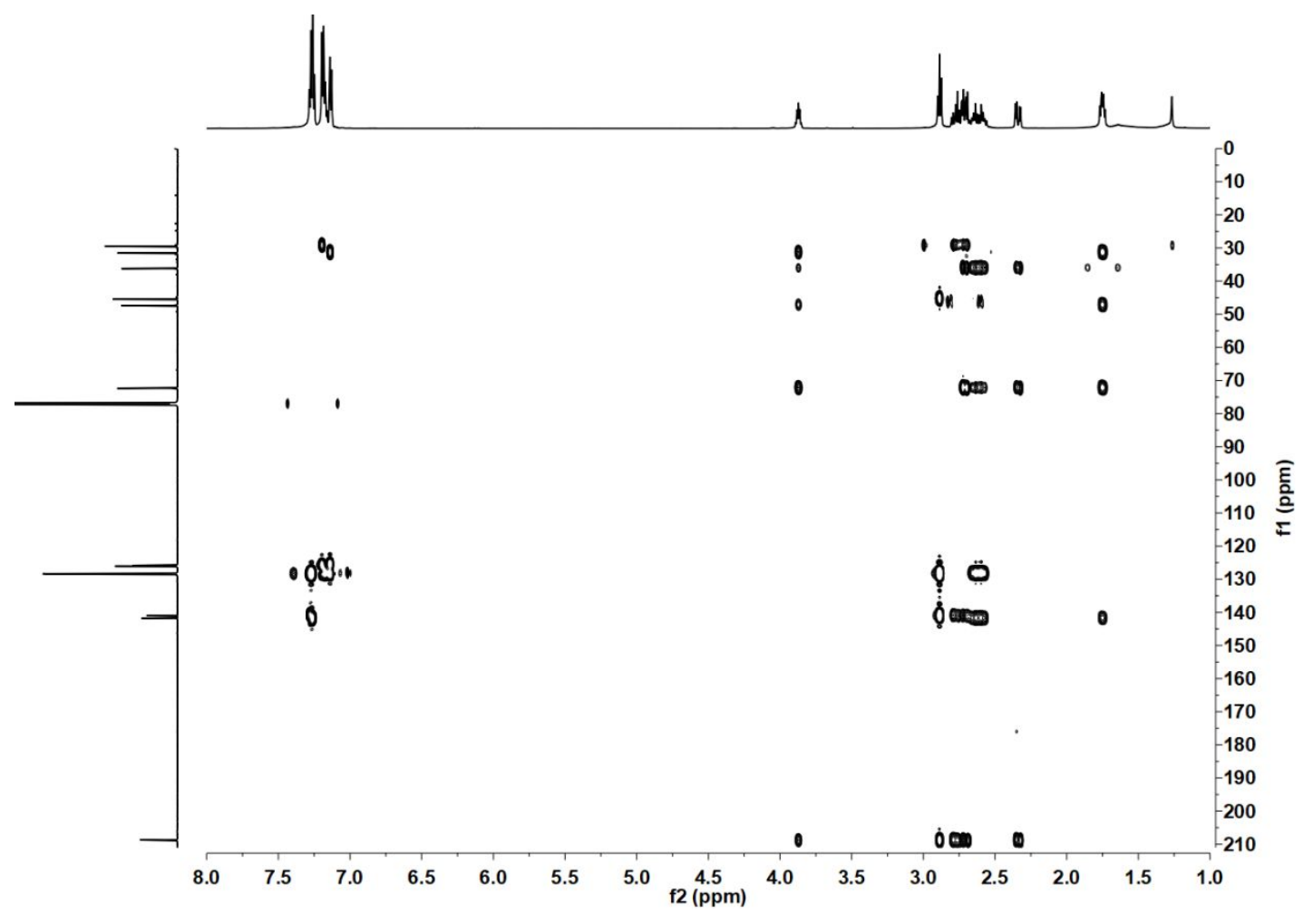

Figure S19. $\mathrm{HMBC}$ spectrum of 2 in $\mathrm{CDCl}_{3}$. 


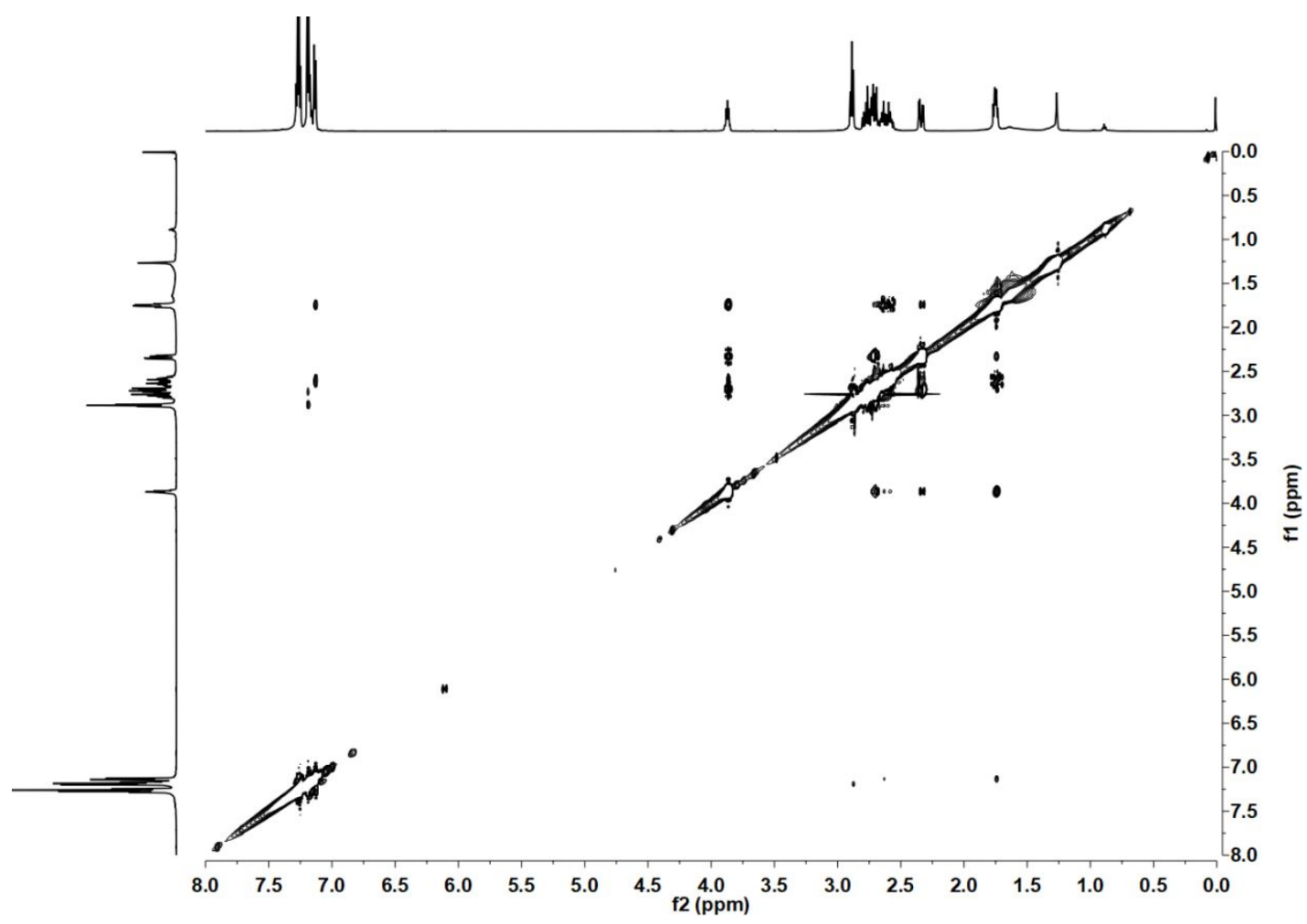

Figure S20. NOESY spectrum of 2 in $\mathrm{CDCl}_{3}$.

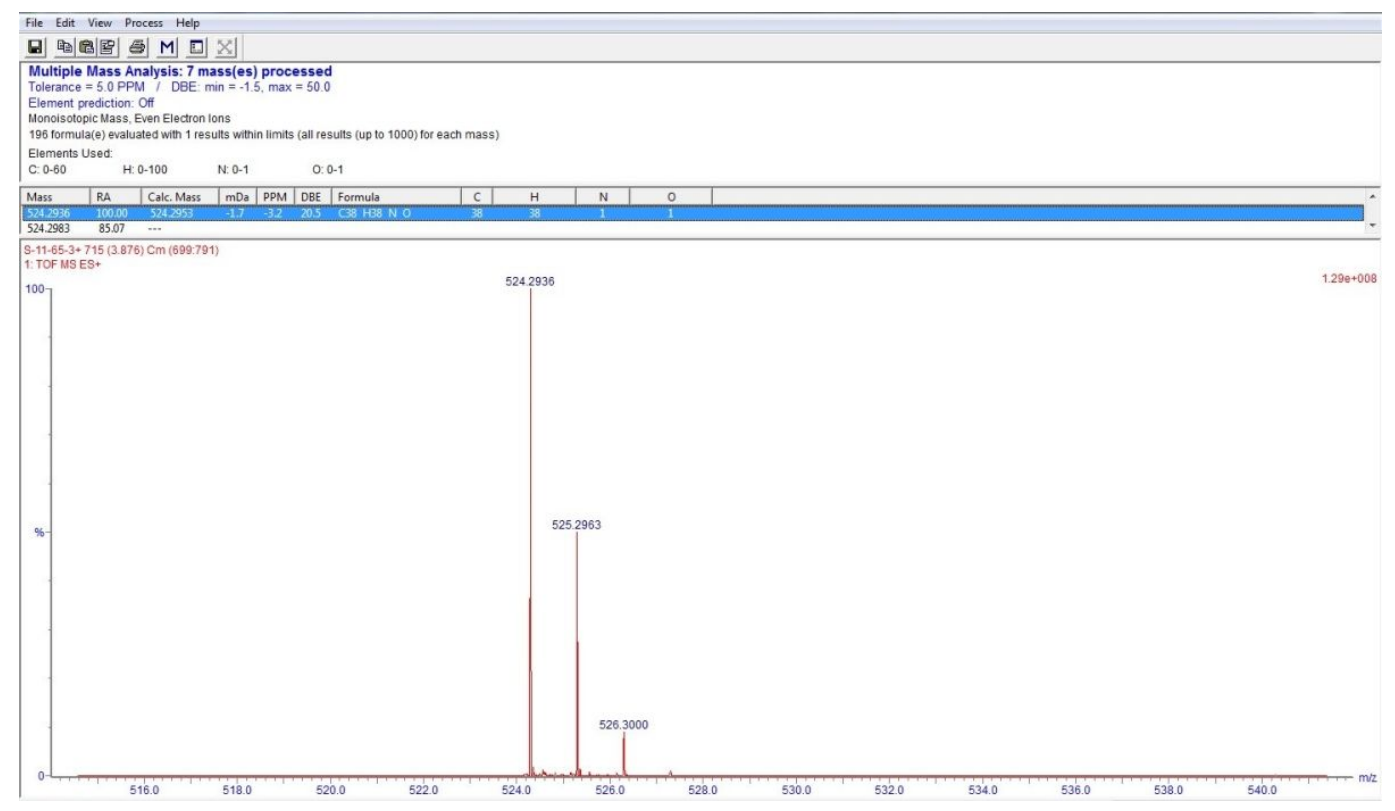

Figure S21. HR-ESI-MS spectrum of 3. 


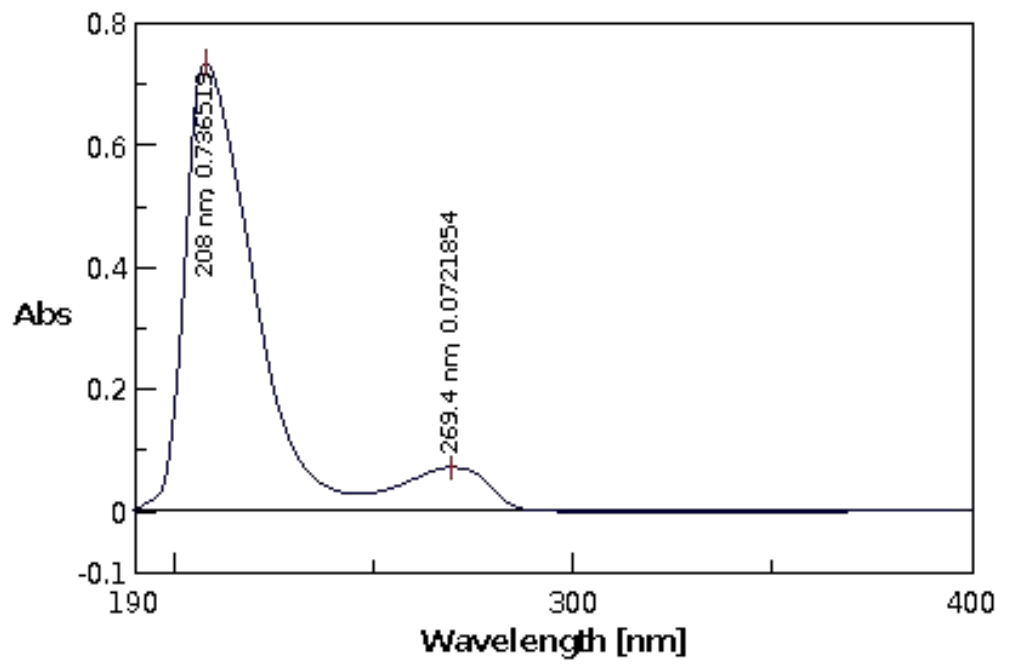

Figure S22. UV spectrum (MeOH) of 3.

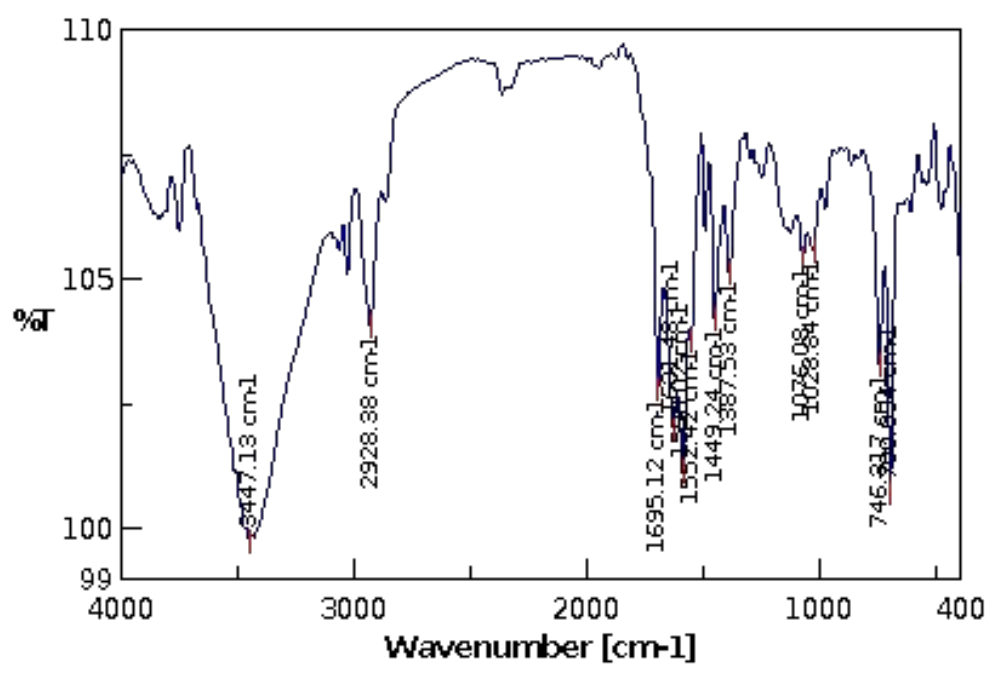

Figure S23. IR spectrum ( $\mathrm{KBr})$ of 3. 


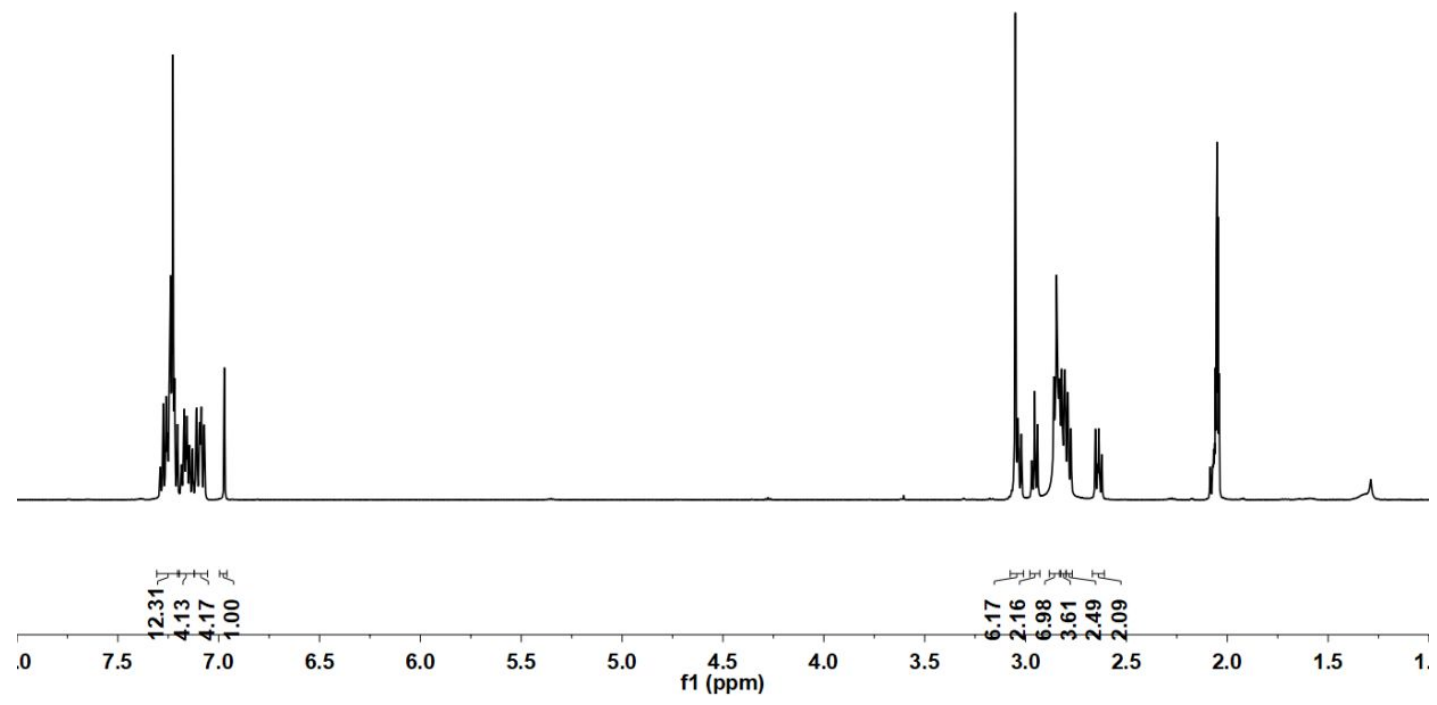

Figure S24. ${ }^{1} \mathrm{H}$ NMR Spectrum of 3 in acetone- $d_{6}(500 \mathrm{MHz})$.

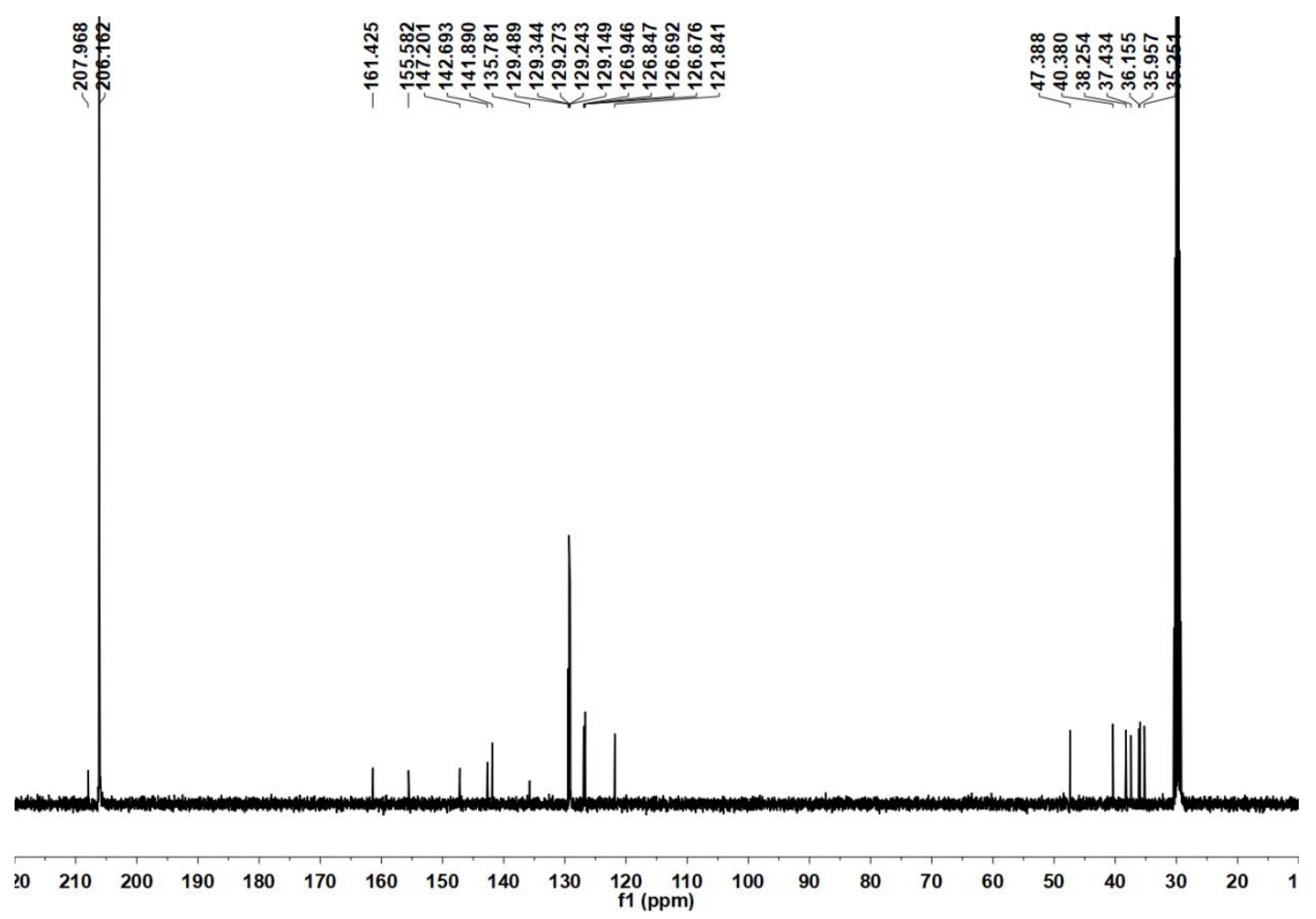

Figure S25. ${ }^{13} \mathrm{C}$ NMR spectrum of $\mathbf{3}$ in acetone- $d_{6}(125 \mathrm{MHz})$. 


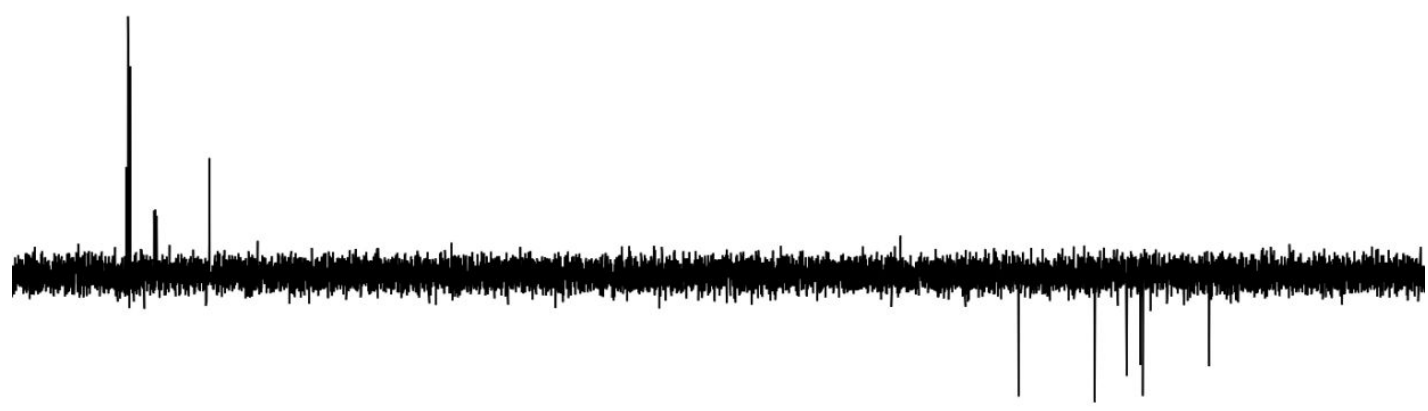

\begin{tabular}{lllllllllllllllllllllllllllllllll}
\hline 0 & 135 & 130 & 125 & 120 & 115 & 110 & 105 & 100 & 95 & 90 & 85 & 80 & $\begin{array}{c}75 \\
\mathrm{f} 1(\mathrm{ppm})\end{array}$ & 70 & 65 & 60 & 55 & 50 & 45 & 40 & 35 & 30 & 25 & 20 & 15 & 1
\end{tabular}

Figure S26. DEPT-135 spectrum of 3 in acetone- $d_{6}(125 \mathrm{MHz})$.

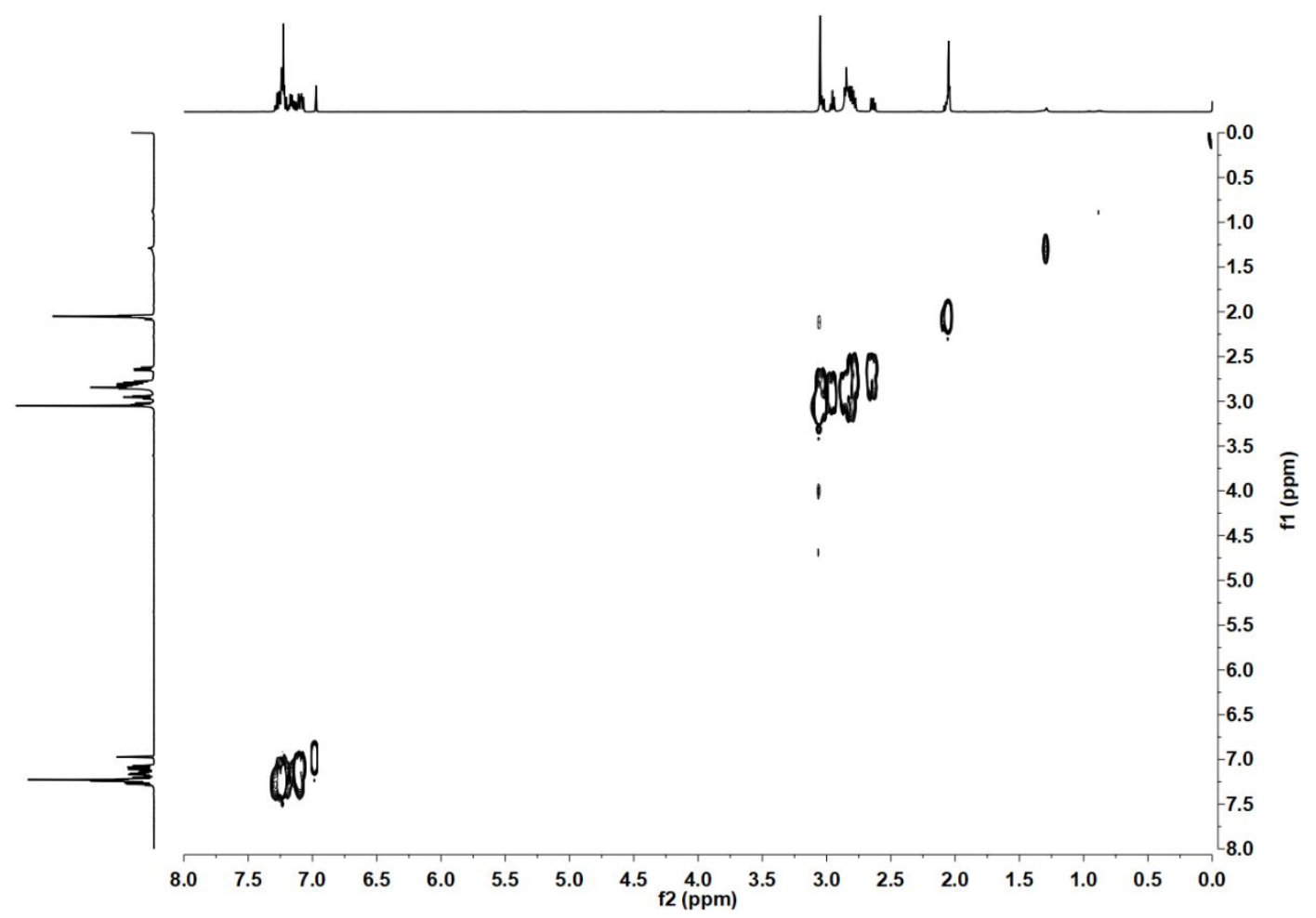

Figure S27. ${ }^{1} \mathrm{H}-{ }^{1} \mathrm{H}$ COSY spectrum of $\mathbf{3}$ in acetone- $d_{6}$. 


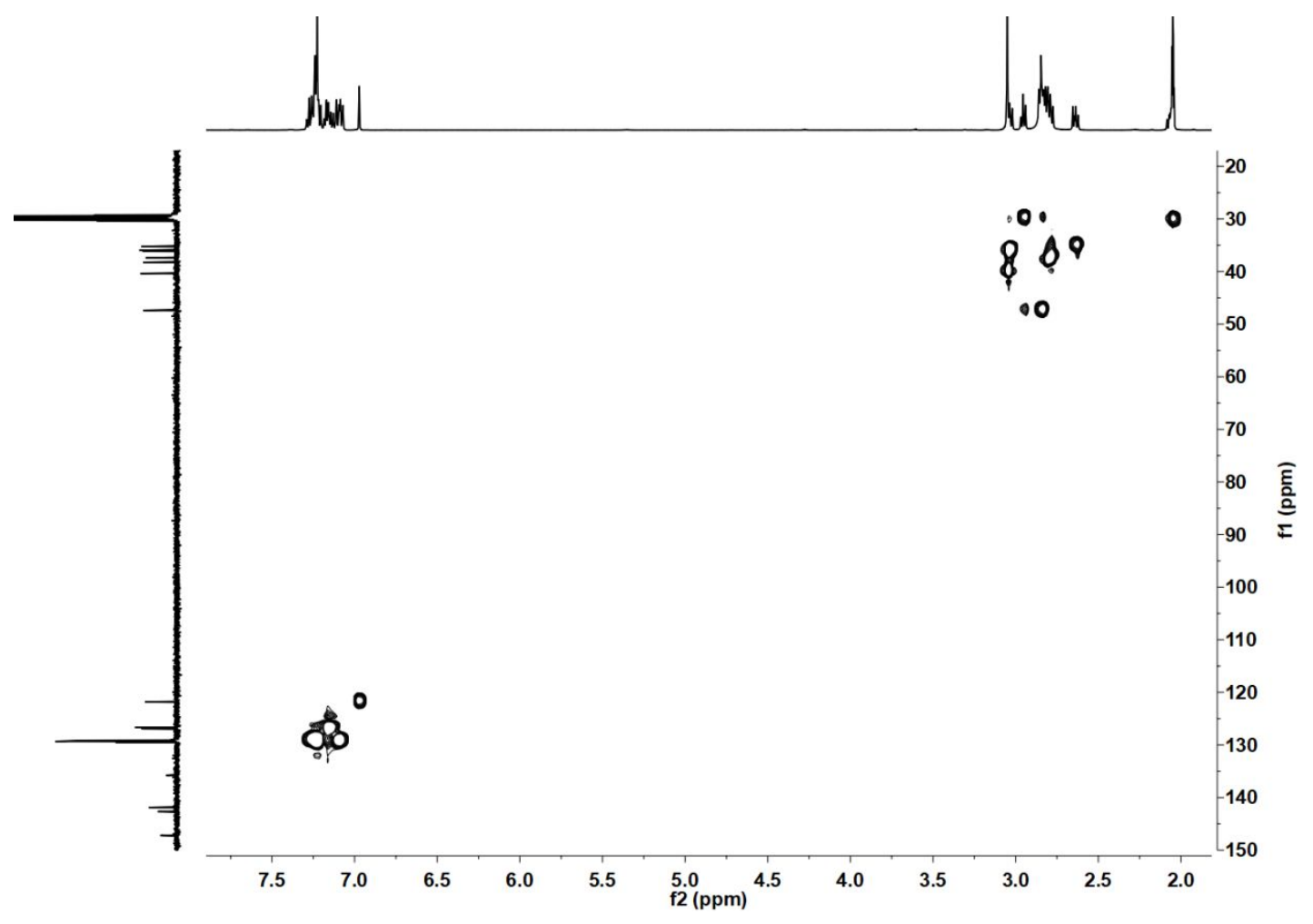

Figure S28. HSQC spectrum of $\mathbf{3}$ in acetone- $d_{6}$.

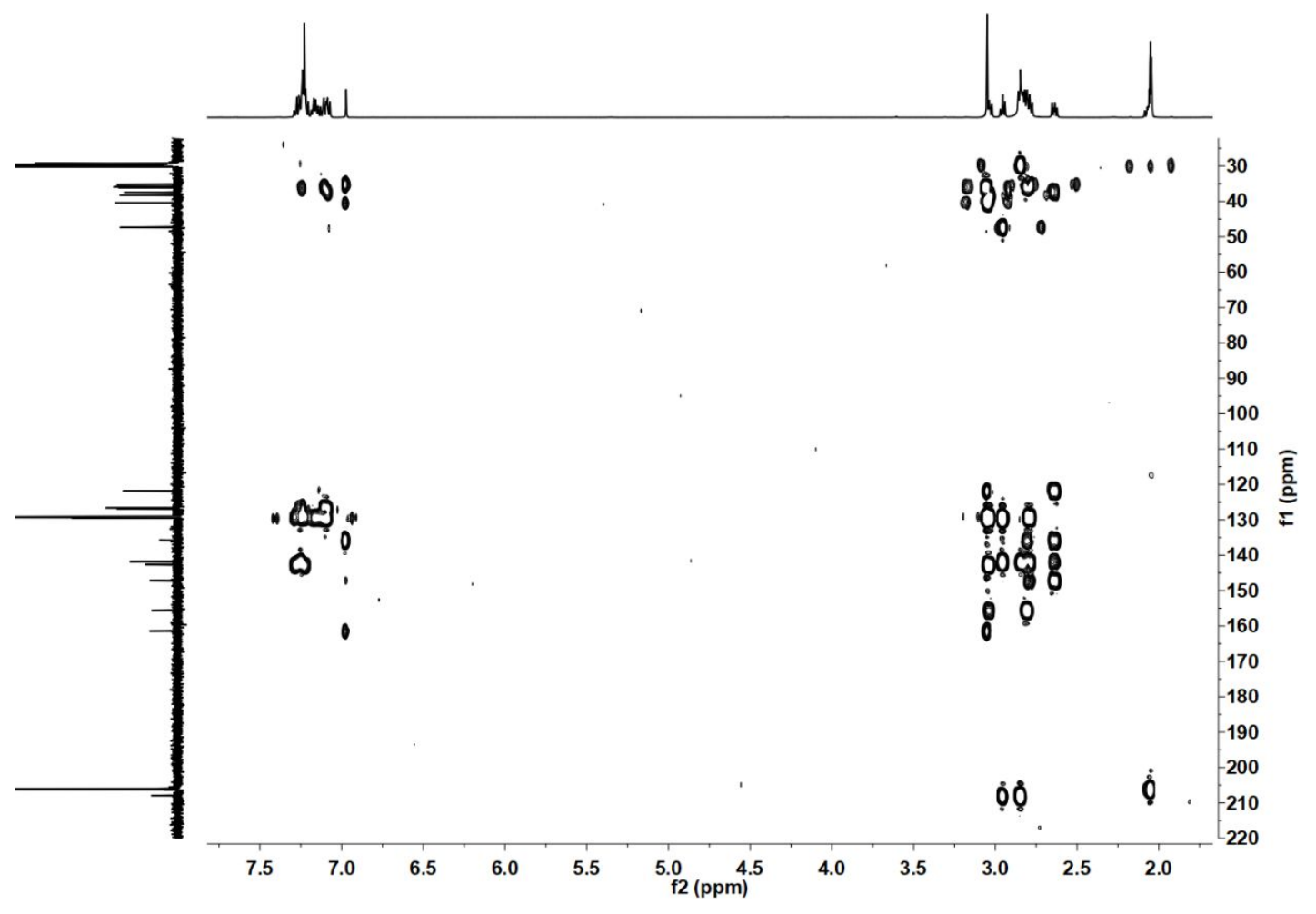

Figure S29. HMBC spectrum of $\mathbf{3}$ in acetone- $d_{6}$. 


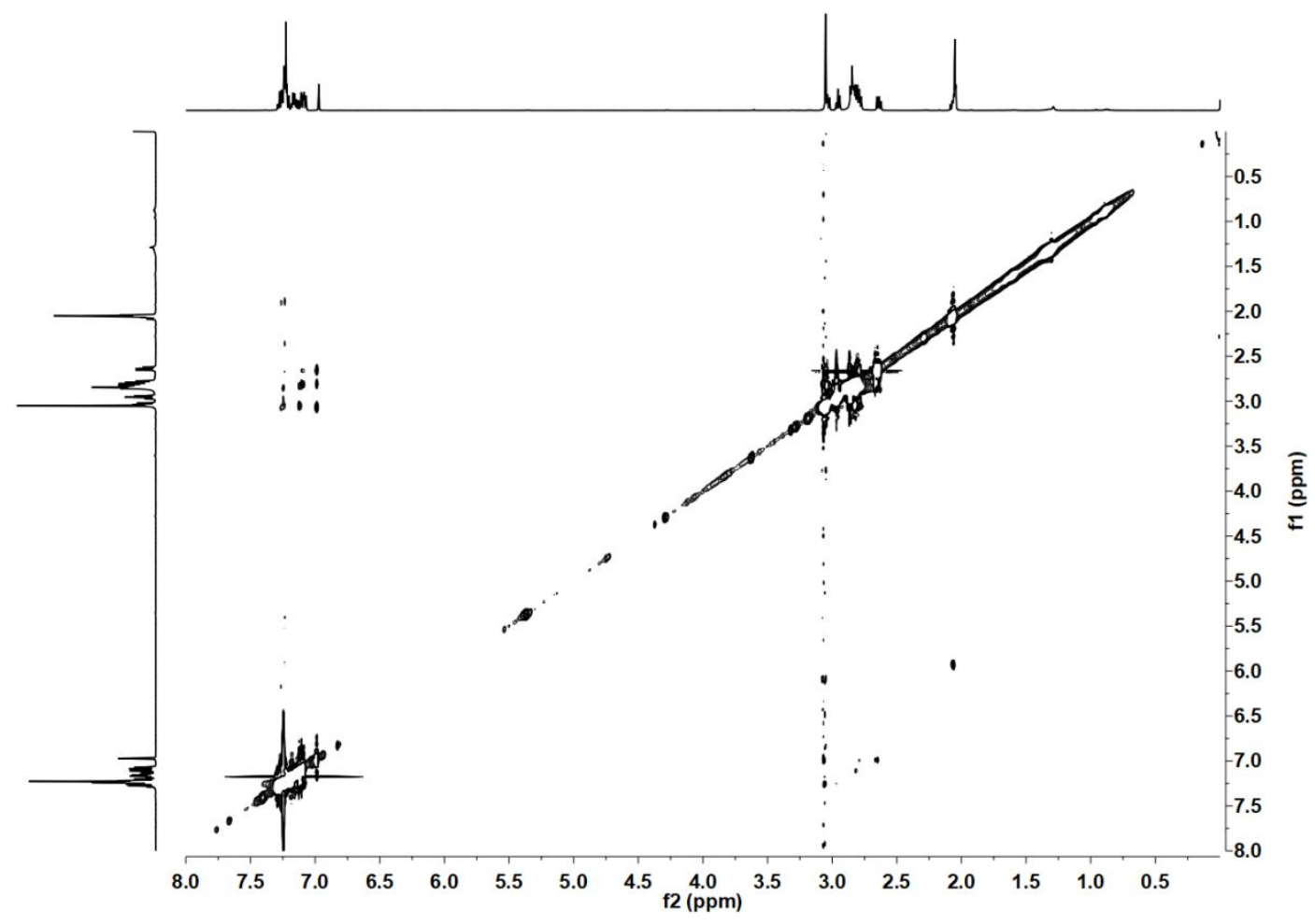

Figure S30. NOESY spectrum of $\mathbf{3}$ in acetone- $d_{6}$.

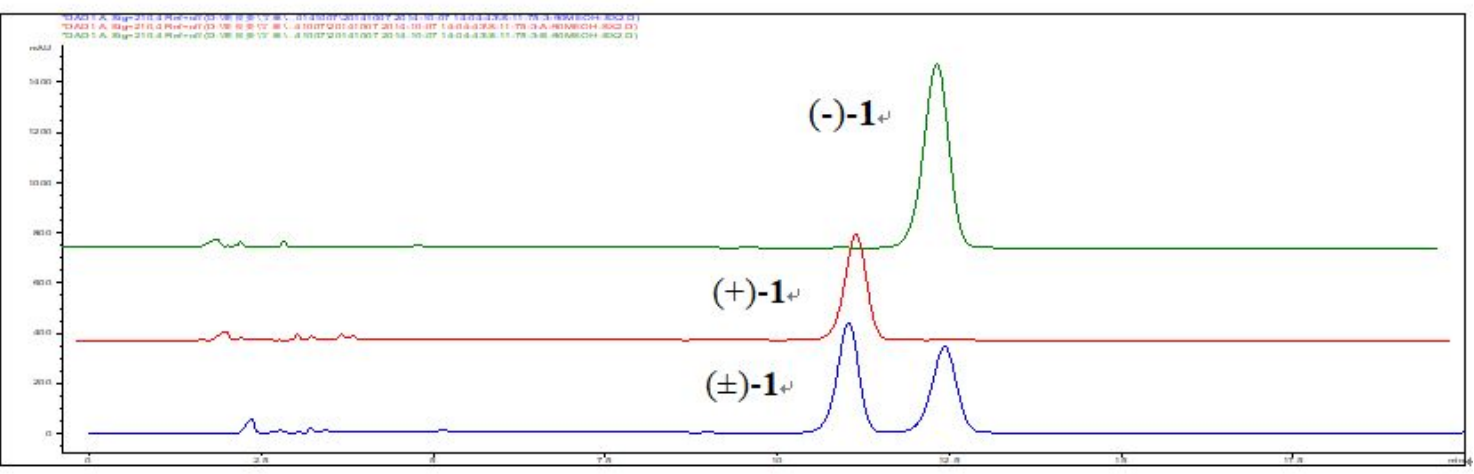

Figure S31. Chiral HPLC profiles of $( \pm)-1$ eluting with the condition: Column: Phenomenex Cellulose-4 (4.6 $\times 250 \mathrm{~mm}, 5 \mu \mathrm{m})$; Mobile phase: $\mathrm{MeOH}-\mathrm{H}_{2} \mathrm{O}, 80: 20$, v/v; Flow: $1 \mathrm{~mL} / \mathrm{min}$; Wavelength: $210 \mathrm{~nm}$. 
Table S1. DP4+ probability analyses of calculated and experimental ${ }^{1} \mathrm{H}$ NMR data of $\mathbf{1}$.

\begin{tabular}{|c|c|c|c|c|c|}
\hline \multirow{2}{*}{ No. } & \multirow{2}{*}{$\delta_{\text {exp }}$} & \multicolumn{2}{|c|}{$\delta_{\text {scal }}$} & \multicolumn{2}{|c|}{ corrected error $(\Delta \delta)$} \\
\hline & & 4R5R3"'S5"'S & $4 R 5 R 3$ "'R5"'S & 4R5R3"'S5"'S & 4R5R3"'R5"'S \\
\hline $1 \mathrm{a}$ & 2.8 & 2.54 & 2.37 & -0.29 & -0.46 \\
\hline $1 b$ & 2.8 & 3.50 & 2.41 & 0.67 & -0.42 \\
\hline $2 \mathrm{a}$ & 2.8 & 2.93 & 1.09 & 0.09 & -1.75 \\
\hline $2 b$ & 2.8 & 2.98 & 1.72 & 0.14 & -1.12 \\
\hline 4 & 2.7 & 2.64 & 2.62 & -0.02 & -0.04 \\
\hline 5 & 3.8 & 3.85 & 3.05 & 0.04 & -0.76 \\
\hline $6 \mathrm{a}$ & 1.7 & 1.47 & 2.09 & -0.22 & 0.40 \\
\hline $6 b$ & 1.4 & 0.85 & 1.77 & -0.52 & 0.40 \\
\hline $7 \mathrm{a}$ & 2.9 & 2.46 & 3.02 & -0.45 & 0.11 \\
\hline $7 b$ & 2.6 & 3.15 & 2.84 & 0.53 & 0.22 \\
\hline $2^{\prime}$ & 7.2 & 7.78 & 6.80 & 0.59 & -0.39 \\
\hline $3^{\prime}$ & 7.2 & 7.40 & 7.22 & 0.21 & 0.03 \\
\hline $4^{\prime}$ & 7.2 & 7.33 & 7.17 & 0.18 & 0.02 \\
\hline $5^{\prime}$ & 7.2 & 7.28 & 7.24 & 0.09 & 0.05 \\
\hline $6^{\prime}$ & 7.2 & 7.04 & 7.11 & -0.15 & -0.08 \\
\hline $2^{\prime \prime}$ & 7.1 & 6.37 & 7.21 & -0.73 & 0.11 \\
\hline $3^{\prime \prime}$ & 7.3 & 7.09 & 7.38 & -0.20 & 0.09 \\
\hline 4" & 7.2 & 7.11 & 7.28 & -0.08 & 0.09 \\
\hline 5" & 7.3 & 7.14 & 7.33 & -0.15 & 0.04 \\
\hline 6" & 7.1 & 7.11 & 7.28 & 0.01 & 0.18 \\
\hline $1 \mathrm{l'a}$ & 2.7 & 2.40 & 3.24 & -0.32 & 0.52 \\
\hline $1 " \mathrm{l} b$ & 2.6 & 2.66 & 2.72 & 0.08 & 0.14 \\
\hline 2 "'a & 1.6 & 1.73 & 1.77 & 0.15 & 0.19 \\
\hline 2"'b & 1.6 & 1.63 & 2.07 & 0.05 & 0.49 \\
\hline 4"'a & 1.8 & 1.32 & 2.43 & -0.46 & 0.65 \\
\hline 4"'b & 1.2 & 1.48 & 1.56 & 0.26 & 0.34 \\
\hline $5 " '$ & 3.9 & 3.93 & 3.36 & 0.07 & -0.50 \\
\hline 6"'"a & 1.9 & 1.69 & 1.93 & -0.16 & 0.08 \\
\hline 6"'b & 1.7 & 1.96 & 1.84 & 0.25 & 0.13 \\
\hline 7"'a & 2.9 & 2.78 & 3.12 & -0.14 & 0.20 \\
\hline 7"'b & 2.7 & 3.12 & 2.77 & 0.40 & 0.05 \\
\hline $2^{\prime \prime \prime \prime}$ & 7.1 & 7.04 & 7.19 & -0.06 & 0.09 \\
\hline 3"'" & 7.3 & 7.21 & 7.35 & -0.06 & 0.08 \\
\hline 4"'" & 7.2 & 7.15 & 7.15 & -0.04 & -0.04 \\
\hline 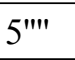 & 7.3 & 7.26 & 7.22 & -0.01 & -0.05 \\
\hline 6"'"' & 7.1 & 7.12 & 7.31 & 0.02 & 0.21 \\
\hline 2"'"' & 7.1 & 7.25 & 7.31 & 0.15 & 0.21 \\
\hline 3"'"' & 7.3 & 7.23 & 7.34 & -0.04 & 0.07 \\
\hline 4"'"' & 7.19 & 7.17 & 7.27 & -0.02 & 0.08 \\
\hline
\end{tabular}




\begin{tabular}{|l|l|l|l|l|l|}
\hline 5 "'"' & 7.27 & 7.30 & 7.36 & 0.03 & 0.09 \\
\hline $6 " '+1$ & 7.10 & 7.20 & 7.33 & 0.10 & 0.23 \\
\hline
\end{tabular}


Table S2. DP4+ probability analyses of calculated and experimental ${ }^{13} \mathrm{C}$ NMR data of $\mathbf{1}$.

\begin{tabular}{|c|c|c|c|c|c|}
\hline \multirow{2}{*}{ No. } & \multirow{2}{*}{$\delta_{\text {exp }}$} & \multicolumn{2}{|c|}{$\delta_{\text {scal }}$} & \multicolumn{2}{|c|}{ corrected error $(\Delta \delta)$} \\
\hline & & 4R5R3"'S5"'S & $4 R 5 R 3$ "'R5"'S & 4R5R3"'S5"'S & $4 R 5 R 3$ "'R5"'S \\
\hline 1 & 28.6 & 32.49 & 33.94 & 28.51 & 29.49 \\
\hline 2 & 49.8 & 55.08 & 51.45 & 50.41 & 46.61 \\
\hline 3 & 216.1 & 229.55 & 220.86 & 219.52 & 212.21 \\
\hline 4 & 61.7 & 62.65 & 62.65 & 57.75 & 57.56 \\
\hline 5 & 74.2 & 79.78 & 79.15 & 74.35 & 73.69 \\
\hline 6 & 36.4 & 43.46 & 46.13 & 39.15 & 41.41 \\
\hline 7 & 32.1 & 38.04 & 35.67 & 33.89 & 31.19 \\
\hline $1^{\prime}$ & 140.4 & 149.19 & 149.79 & 141.63 & 142.74 \\
\hline $2^{\prime}$ & 128.5 & 138.89 & 135.57 & 131.64 & 128.84 \\
\hline $3^{\prime}$ & 128.6 & 135.12 & 134.70 & 127.99 & 127.99 \\
\hline $4^{\prime}$ & 126.1 & 132.73 & 132.20 & 125.67 & 125.55 \\
\hline $5^{\prime}$ & 128.6 & 135.73 & 134.78 & 128.58 & 128.06 \\
\hline 61 & 128.5 & 135.74 & 135.95 & 128.59 & 129.21 \\
\hline $1 "$ & 141.8 & 149.80 & 149.54 & 142.22 & 142.49 \\
\hline $2^{\prime \prime}$ & 128.6 & 136.04 & 135.46 & 128.89 & 128.74 \\
\hline 3" & 129.3 & 134.69 & 135.60 & 127.57 & 128.87 \\
\hline 4" & 126.4 & 132.03 & 132.62 & 124.99 & 125.95 \\
\hline $5^{\prime \prime}$ & 129.3 & 134.67 & 135.22 & 127.56 & 128.49 \\
\hline 6" & 128.6 & 135.07 & 136.48 & 127.94 & 129.73 \\
\hline $1 " '$ & 29.4 & 37.33 & 33.65 & 33.20 & 29.21 \\
\hline 2"' & 44.6 & 50.47 & 43.10 & 45.94 & 38.45 \\
\hline $3 " '$ & 72.4 & 77.87 & 78.29 & 72.50 & 72.84 \\
\hline 4"'" & 40.8 & 43.32 & 43.67 & 39.01 & 39.00 \\
\hline $5 " '$ & 72.2 & 72.51 & 78.74 & 67.30 & 73.29 \\
\hline 6"' & 37.8 & 42.92 & 46.25 & 38.62 & 41.52 \\
\hline 7"' & 32.0 & 35.89 & 37.73 & 31.81 & 33.20 \\
\hline $1 " '+$ & 142.3 & 150.21 & 152.53 & 142.61 & 145.42 \\
\hline $2^{\prime \prime \prime \prime}$ & 128.5 & 134.75 & 136.23 & 127.63 & 129.49 \\
\hline $3^{\prime \prime \prime \prime}$ & 128.6 & 135.31 & 135.28 & 128.17 & 128.56 \\
\hline 4"'"' & 126.0 & 132.60 & 132.46 & 125.54 & 125.80 \\
\hline $5^{\prime \prime \prime \prime}$ & 128.6 & 135.64 & 135.19 & 128.49 & 128.46 \\
\hline $6 "$ 6"' & 128.5 & 135.56 & 136.47 & 128.41 & 129.72 \\
\hline 1 "'"' & 142.1 & 149.71 & 150.10 & 142.13 & 143.04 \\
\hline 2"'"' & 128.5 & 136.90 & 135.62 & 129.72 & 128.89 \\
\hline 3"'"' & 128.6 & 134.93 & 135.31 & 127.80 & 128.59 \\
\hline 4"'"' & 125.9 & 132.56 & 132.51 & 125.51 & 125.85 \\
\hline $5^{\prime \prime \prime \prime \prime}$ & 128.6 & 135.36 & 135.41 & 128.22 & 128.68 \\
\hline $6^{\prime \prime \prime \prime \prime}$ & 128.5 & 135.18 & 135.56 & 128.05 & 128.83 \\
\hline
\end{tabular}




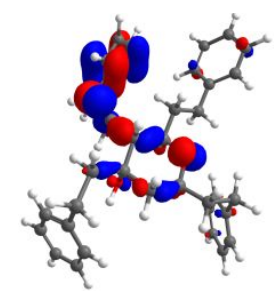

MO 143

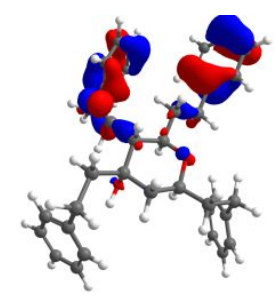

MO 146

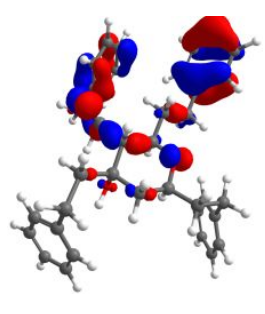

HOMO 147

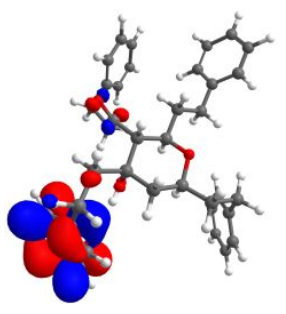

LUMO 148

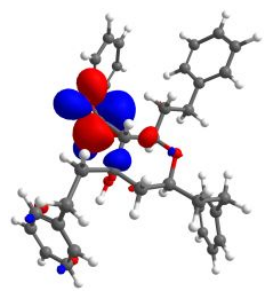

MO 149

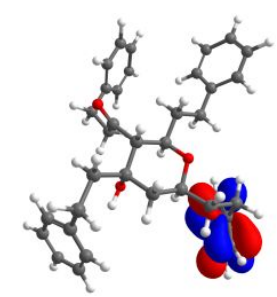

MO 152

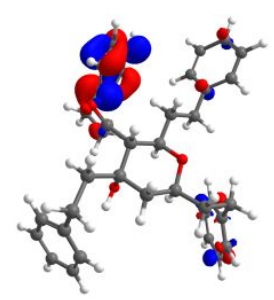

MO 153

Figure S32. Key molecular orbitals involved in important transitions regarding the ECD spectrum of the dominant conformer of $\mathbf{1}$ 
Table S3. Key transitions and their related rotatory and oscillator strengths of the dominant conformer of 1

\begin{tabular}{|c|c|c|c|c|c|}
\hline \multicolumn{6}{|c|}{ HOMO is 147} \\
\hline No. & \begin{tabular}{|l|} 
Energy \\
$\left(\mathrm{cm}^{-1}\right)$
\end{tabular} & $\begin{array}{l}\text { Wavelength } \\
(\mathrm{nm})\end{array}$ & $\begin{array}{l}\text { R } \\
\text { (length) }\end{array}$ & $\begin{array}{l}\text { Osc. } \\
\text { strength }\end{array}$ & Major contribs \\
\hline 1 & 34127.97 & 293.01 & -9.9771 & 0.0006 & $\begin{array}{l}\mathrm{H}-4->\mathrm{L}+1 \quad(24 \%), \quad \mathrm{H}-1->\mathrm{L}+1 \quad(17 \%), \\
\text { HOMO->L+1 }(23 \%)\end{array}$ \\
\hline 2 & 41400.72 & 241.54 & -0.3970 & 0.0001 & $\begin{array}{l}\text { H-4->L+1 }(31 \%), \quad H-1->L+1 \quad(28 \%), \\
\text { HOMO->L+1 }(20 \%)\end{array}$ \\
\hline 3 & 42194.38 & 237.00 & 5.2931 & 0.0042 & H-1->L+1 (40\%), HOMO->L+1 (44\%) \\
\hline 4 & 42230.68 & 236.79 & -1.9657 & 0.0018 & $\begin{array}{l}\text { H-9->LUMO }(20 \%), \mathrm{H}-8->\mathrm{L}+2(12 \%), \\
\text { H-7->LUMO }(18 \%), \mathrm{H}-7->\mathrm{L}+2(25 \%)\end{array}$ \\
\hline 5 & 42612.18 & 234.67 & 0.5736 & 0.0009 & H-6->L+3 (34\%), H-2->L+4 (40\%) \\
\hline 6 & 42625.08 & 234.60 & 1.5427 & 0.0006 & $\begin{array}{l}\text { H-1->LUMO (20\%), HOMO->LUMO } \\
(46 \%)\end{array}$ \\
\hline 7 & 42671.86 & 234.35 & -0.2387 & 0.0000 & H-3->L+6 $(10 \%), H-1->L+8(10 \%)$ \\
\hline 8 & 42734.78 & 234.00 & -1.0981 & 0.0005 & $\begin{array}{l}\text { H-5->L+5 }(14 \%), \quad H-1->L+9 \quad(10 \%), \\
\text { HOMO->L+7 }(10 \%), \quad \text { HOMO->L+8 } \\
(11 \%)\end{array}$ \\
\hline 9 & 43501.01 & 229.88 & -2.6059 & 0.0006 & $\begin{array}{l}\mathrm{H}-5->\mathrm{L}+1 \quad(21 \%), \quad \mathrm{H}-1->\mathrm{L}+2 \quad(16 \%), \\
\text { HOMO->L+2 }(34 \%)\end{array}$ \\
\hline 10 & 43511.49 & 229.82 & 3.0007 & 0.0022 & 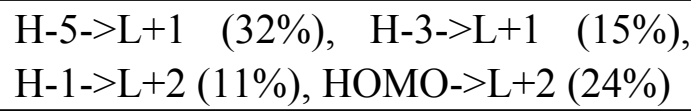 \\
\hline 11 & 43915.58 & 227.71 & 0.1805 & 0.0019 & $\begin{array}{l}\text { H-1->LUMO (65\%), HOMO->LUMO } \\
(26 \%)\end{array}$ \\
\hline 12 & 44255.95 & 225.96 & 14.3155 & 0.0106 & $\begin{array}{l}\mathrm{H}-7->\mathrm{L}+1 \quad(39 \%), \quad \mathrm{H}-4->\mathrm{L}+1 \quad(12 \%), \\
\mathrm{H}-2->\mathrm{L}+1 \quad(14 \%)\end{array}$ \\
\hline 13 & 44531.79 & 224.56 & 0.4587 & 0.0021 & H-2->LUMO (79\%) \\
\hline 14 & 44614.06 & 224.14 & -2.0646 & 0.0009 & $\begin{array}{l}\text { H-5->L+1 }(16 \%), \quad H-3->L+1 \quad(11 \%), \\
\text { H-2->LUMO }(11 \%), H-2->L+1(49 \%)\end{array}$ \\
\hline 15 & 44643.10 & 224.00 & -3.1906 & 0.0019 & $\begin{array}{l}\text { H-5->L+1 }(10 \%), \quad \text { H-3->L+1 }(26 \%), \\
\text { H-1->L+2 }(35 \%), \text { HOMO->L+2 }(14 \%)\end{array}$ \\
\hline 16 & 44649.55 & 223.97 & 1.9067 & 0.0030 & $\begin{array}{l}\text { H-3->L+1 }(23 \%), \quad \text { H-2->L+1 } \quad(16 \%), \\
\text { H-1->L+2 }(32 \%), \text { HOMO->L+2 }\end{array}$ \\
\hline 17 & 44972.98 & 222.36 & 1.1038 & 0.0008 & $\begin{array}{l}\begin{array}{l}\text { H-4->LUMO } \\
(23 \%)\end{array} \\
(56 \%), \quad \text { H-3->LUMO }\end{array}$ \\
\hline 18 & 45260.92 & 220.94 & -0.0206 & 0.0000 & $\mathrm{H}-2->\mathrm{L}+2(96 \%)$ \\
\hline 19 & 45732.76 & 218.66 & -1.1866 & 0.0007 & $\begin{array}{lll}\text { H-4->LUMO } & (24 \%), & \text { H-3->LUMO } \\
(54 \%) & \end{array}$ \\
\hline 20 & 45771.47 & 218.48 & -2.6077 & 0.0012 & $\mathrm{H}-4->\mathrm{L}+2(49 \%), \mathrm{H}-3->\mathrm{L}+2(22 \%)$ \\
\hline 21 & 45796.48 & 218.36 & -46.2718 & 0.0404 & $\begin{array}{l}\text { H-1->L+5 (15\%), HOMO->L+4 (10\%), } \\
\text { HOMO->L+5 }(44 \%)\end{array}$ \\
\hline
\end{tabular}




\begin{tabular}{|c|c|c|c|c|c|}
\hline 22 & 46115.07 & 216.85 & 11.4235 & 0.0050 & HOMO->L+3 (76\%) \\
\hline 23 & 46190.08 & 216.50 & -3.5363 & 0.0052 & H-5->LUMO (75\%) \\
\hline 24 & 46256.22 & 216.19 & 16.2071 & 0.0065 & $\begin{array}{l}\mathrm{H}-1->\mathrm{L}+5(40 \%), \text { HOMO->L+5 }(19 \%), \\
\text { HOMO->L+6 }(15 \%)\end{array}$ \\
\hline 25 & 46383.65 & 215.59 & -7.3728 & 0.0309 & $\begin{array}{l}\text { H-7->LUMO }(23 \%), \text { H-7->L+2 }(12 \%), \\
\text { H-6->LUMO } \quad(26 \%), \quad \text { H-5->LUMO } \\
(10 \%)\end{array}$ \\
\hline 26 & 46406.24 & 215.49 & -7.0425 & 0.0030 & $\begin{array}{l}\text { H-1->L+7 (16\%), HOMO->L+4 (17\%), } \\
\text { HOMO->L+6 (31\%) }\end{array}$ \\
\hline 27 & 46486.09 & 215.12 & -3.0013 & 0.0022 & H-1->L+6 (44\%), HOMO->L+7 (16\%) \\
\hline 28 & 46499.80 & 215.05 & 2.9284 & 0.0022 & $\begin{array}{l}\text { H-6->LUMO (31\%), H-4->L+2 (15\%), } \\
\text { H-3->L+2 }(31 \%)\end{array}$ \\
\hline 29 & 46507.86 & 215.02 & 2.5879 & 0.0003 & $\begin{array}{l}\text { HOMO- }>\text { L }+4 \quad(40 \%), \quad \text { HOMO- }>\mathrm{L}+6 \\
(13 \%), \text { HOMO->L }+7(15 \%)\end{array}$ \\
\hline 30 & 46532.06 & 214.91 & -1.2209 & 0.0060 & $\begin{array}{l}\text { H-7->LUMO }(14 \%), \quad \text { H-6->LUMO } \\
(13 \%), \quad \text { H-6->L+1 }(20 \%), \quad \text { H-4->L+2 } \\
(14 \%), H-3->L+2(21 \%)\end{array}$ \\
\hline 31 & 46590.94 & 214.63 & -4.1228 & 0.0037 & $\begin{array}{l}\text { H-7->L+1 }(10 \%), \text { H-6->LUMO }(23 \%), \\
\text { H-6->L+1 }(54 \%)\end{array}$ \\
\hline 32 & 46650.62 & 214.36 & 1.5068 & 0.0071 & $\mathrm{H}-1->\mathrm{L}+3(62 \%)$ \\
\hline 33 & 46835.33 & 213.51 & 10.6177 & 0.0124 & 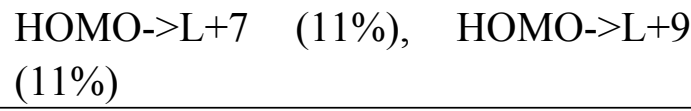 \\
\hline 34 & 46968.41 & 212.91 & 4.1138 & 0.0218 & $\mathrm{H}-2->\mathrm{L}+3(25 \%), \mathrm{H}-1->\mathrm{L}+3(17 \%)$ \\
\hline 35 & 46989.38 & 212.81 & 0.5378 & 0.0014 & $\mathrm{H}-5->\mathrm{L}+2(86 \%)$ \\
\hline 36 & 47022.45 & 212.66 & -14.6334 & 0.0107 & $\mathrm{H}-1->\mathrm{L}+4(47 \%)$ \\
\hline 37 & 47044.23 & 212.57 & 0.8136 & 0.0028 & $\begin{array}{l}\text { H-9->L+1 }(17 \%), \quad H-8->L+1 \quad(35 \%), \\
H-7->L+1(18 \%), H-6->L+1(10 \%)\end{array}$ \\
\hline 38 & 47204.73 & 211.84 & 0.9478 & 0.0001 & $\mathrm{H}-6->\mathrm{L}+2(94 \%)$ \\
\hline 39 & 47306.36 & 211.39 & -1.5507 & 0.0007 & $\begin{array}{l}\mathrm{H}-4->\mathrm{L}+7 \quad(17 \%), \quad \mathrm{H}-1->\mathrm{L}+7 \quad(17 \%), \\
\mathrm{HOMO}->\mathrm{L}+8(14 \%)\end{array}$ \\
\hline 40 & 47397.50 & 210.98 & -9.5613 & 0.0042 & $\begin{array}{l}\mathrm{H}-4->\mathrm{L}+6 \quad(10 \%), \quad \mathrm{H}-4->\mathrm{L}+7 \quad(11 \%), \\
\mathrm{H}-1->\mathrm{L}+8(13 \%), \text { HOMO->L+8 }\end{array}$ \\
\hline 41 & 47516.06 & 210.46 & 2.3465 & 0.0051 & H-1->L+9 (18\%), HOMO->L+9 (23\%) \\
\hline 42 & 47657.21 & 209.83 & 16.9223 & 0.0066 & $\begin{array}{l}\mathrm{H}-4->\mathrm{L}+5 \quad(16 \%), \quad \mathrm{H}-3->\mathrm{L}+5 \quad(10 \%), \\
\mathrm{H}-2->\mathrm{L}+6(11 \%), \mathrm{H}-2->\mathrm{L}+7(14 \%)\end{array}$ \\
\hline 43 & 47669.31 & 209.78 & -5.7022 & 0.0030 & HOMO->L+9 $(17 \%)$ \\
\hline 44 & 48064.52 & 208.05 & -3.5113 & 0.0020 & 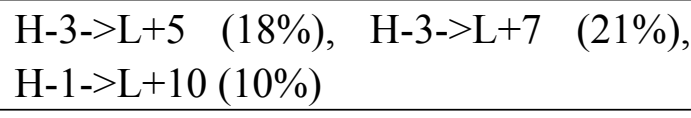 \\
\hline 45 & 48158.08 & 207.65 & -1.3131 & 0.0002 & $\mathrm{H}-3->\mathrm{L}+3(43 \%)$ \\
\hline 46 & 48223.42 & 207.37 & -1.7918 & 0.0043 & $\begin{array}{lll}\mathrm{H}-3->\mathrm{L}+3 & (13 \%), & \text { HOMO- }>\mathrm{L}+10 \\
(10 \%) & & \\
\end{array}$ \\
\hline 47 & 48277.46 & 207.14 & -6.3767 & 0.0030 & $\begin{array}{l}\text { H-9->LUMO } \quad(21 \%), \quad \text { H-8->LUMO } \\
(25 \%), \quad \text { H-8->L+2 } \quad(14 \%), \quad \text { H-7->L+2 }\end{array}$ \\
\hline
\end{tabular}




\begin{tabular}{|c|c|c|c|c|c|}
\hline & & & & & $(10 \%)$ \\
\hline 48 & 48316.98 & 206.97 & -8.8258 & 0.0011 & H-8->LUMO (10\%), H-3->L+5 (10\%) \\
\hline 49 & 48388.76 & 206.66 & 8.2065 & 0.0042 & 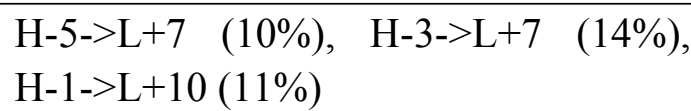 \\
\hline 50 & 48453.29 & 206.38 & 10.8533 & 0.0119 & H-3->L+7 (11\%), H-3->L+9 (11\%) \\
\hline 51 & 48480.71 & 206.27 & -11.0383 & 0.0046 & H-9->L+1 (38\%), H-8->L+1 (18\%) \\
\hline 52 & 48499.26 & 206.19 & -6.7123 & 0.0059 & $\mathrm{H}-3->\mathrm{L}+4(33 \%)$ \\
\hline 53 & 48571.85 & 205.88 & -6.7636 & 0.0099 & $\begin{array}{l}\mathrm{H}-5->\mathrm{L}+6 \quad(22 \%), \quad \mathrm{H}-5->\mathrm{L}+8 \quad(12 \%) \\
\mathrm{H}-4->\mathrm{L}+4(10 \%)\end{array}$ \\
\hline 54 & 48671.86 & 205.46 & -2.3969 & 0.0017 & H-4->L+3 (43\%), H-3->L+3 (21\%) \\
\hline 55 & 48754.94 & 205.11 & 6.1758 & 0.0021 & $\begin{array}{l}\mathrm{H}-2->\mathrm{L}+5 \quad(21 \%), \quad \mathrm{H}-2->\mathrm{L}+6 \quad(19 \%), \\
\mathrm{H}-2->\mathrm{L}+9(10 \%)\end{array}$ \\
\hline 56 & 48796.07 & 204.93 & 6.6041 & 0.0031 & $\begin{array}{l}\text { H-9->L+2 (13\%), H-8->LUMO (10\%), } \\
\text { H-8->L+2 (17\%) }\end{array}$ \\
\hline 57 & 48917.86 & 204.42 & 15.8053 & 0.0045 & H-9->L+2 (11\%), H-4->L+6 (19\%) \\
\hline 58 & 48942.87 & 204.32 & -0.8532 & 0.0008 & $\mathrm{H}-4->\mathrm{L}+4(18 \%)$ \\
\hline 59 & 49032.40 & 203.95 & 2.4403 & 0.0027 & H-7->L+5 (10\%), H-4->L+4 (22\%) \\
\hline 60 & 49128.38 & 203.55 & -3.4850 & 0.0008 & 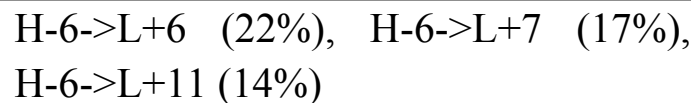 \\
\hline
\end{tabular}




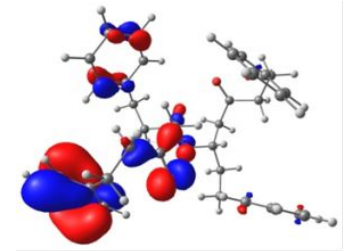

MO 141

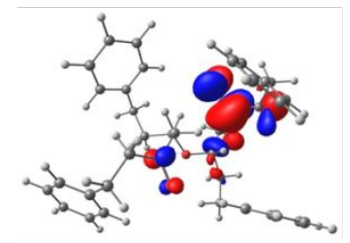

LUMO 148

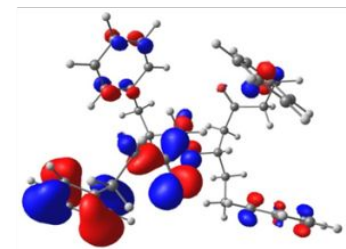

MO 142

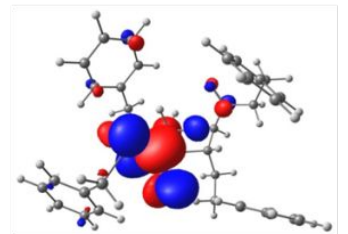

MO 149

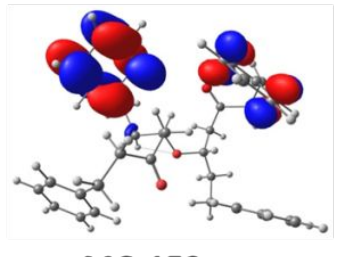

MO 152

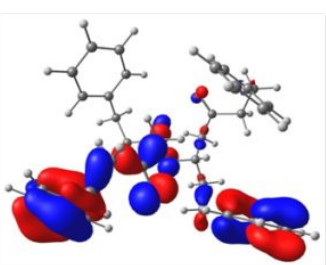

MO 146

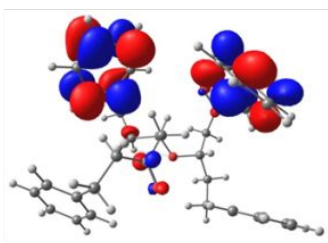

MO 150

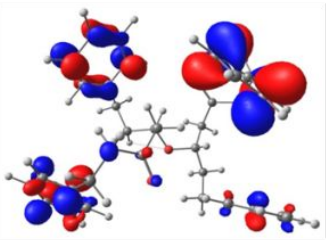

MO 153

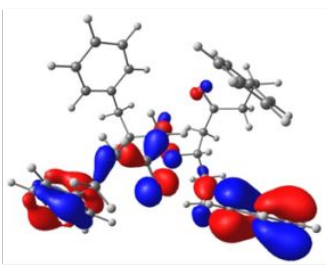

HOMO 147

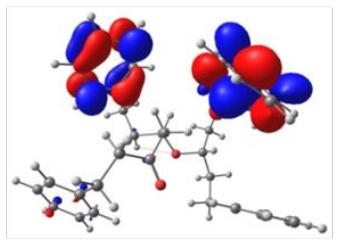

MO 151

Figure S33. Key molecular orbitals involved in important transitions regarding the ECD spectrum of the dominant conformer of 2 
Table S4. Key transitions and their related rotatory and oscillator strengths of the dominant conformer of 2

\begin{tabular}{|c|c|c|c|c|c|}
\hline \multicolumn{6}{|c|}{ HOMO is 147} \\
\hline No. & $\begin{array}{l}\text { Energy } \\
\left(\mathrm{cm}^{-1}\right)\end{array}$ & $\begin{array}{l}\text { Wavelength } \\
(\mathrm{nm})\end{array}$ & $\begin{array}{l}\text { R } \\
\text { (length) }\end{array}$ & $\begin{array}{l}\text { Osc. } \\
\text { strength }\end{array}$ & Major contribs \\
\hline 1 & $\begin{array}{l}33902 . \\
94\end{array}$ & 294.9596437 & 9.2778 & 0.0004 & $\begin{array}{lc}\text { H-6->L+1 } & (12 \%), \text { H-5->L+1 }(20 \%), \\
\text { H-1->L+1 } & (27 \%), \quad \text { HOMO->L+1 } \\
(18 \%) & \end{array}$ \\
\hline 2 & $\begin{array}{l}34540 . \\
93\end{array}$ & 289.5115858 & -10.6804 & 0.0007 & $\begin{array}{ll}\text { H-9->LUMO } & (62 \%), \\
(13 \%) & \text { H-2->LUMO }\end{array}$ \\
\hline 3 & $\begin{array}{l}40023 . \\
93\end{array}$ & 249.8505464 & -2.4127 & 0.0033 & $\begin{array}{l}\text { H-2->LUMO } \quad(19 \%), \quad \text { H-1->LUMO } \\
(13 \%), \text { HOMO->LUMO }(57 \%)\end{array}$ \\
\hline 4 & $\begin{array}{l}40576 . \\
42\end{array}$ & 246.4485502 & 4.2105 & 0.0031 & $\begin{array}{l}\text { H-1->LUMO (60\%), HOMO->LUMO } \\
(26 \%)\end{array}$ \\
\hline 5 & $\begin{array}{l}41026 . \\
48\end{array}$ & 243.7450097 & -3.6306 & 0.0016 & $\begin{array}{l}\text { H-9->LUMO }(17 \%), \quad \text { H-3->LUMO } \\
(16 \%), \text { H-2->LUMO }(45 \%)\end{array}$ \\
\hline 6 & $\begin{array}{l}41399 . \\
11\end{array}$ & 241.5510767 & 7.2468 & 0.0016 & $\begin{array}{ll}\text { H-5->L+1 } & (18 \%), \text { H-2->L+1 }(11 \%), \\
\text { H-1->L+1 } & (26 \%), \quad \text { HOMO->L+1 } \\
(12 \%) & \end{array}$ \\
\hline 7 & $\begin{array}{l}41883 . \\
05\end{array}$ & 238.7600844 & -1.7344 & 0.0004 & $\begin{array}{lll}\mathrm{H}-1->\mathrm{L}+1 & (31 \%), & \text { HOMO- }>\mathrm{L}+1 \\
(62 \%) & \end{array}$ \\
\hline 8 & $\begin{array}{l}41965 . \\
32\end{array}$ & 238.2920174 & -0.8192 & 0.0002 & $\begin{array}{l}\text { H-7->LUMO }(25 \%), \quad \text { H-4->LUMO } \\
(31 \%), \text { H-3->LUMO }(13 \%)\end{array}$ \\
\hline 9 & $\begin{array}{l}42137 . \\
11\end{array}$ & 237.3204767 & -1.079 & 0.0012 & $\begin{array}{l}\text { H-4->LUMO }(17 \%), \quad \text { H-3->LUMO } \\
(12 \%)\end{array}$ \\
\hline 10 & $\begin{array}{l}42270 . \\
2\end{array}$ & 236.573303 & 0.4303 & 0.0007 & $\begin{array}{l}\text { H-7->LUMO }(15 \%), \quad \text { H-3->LUMO } \\
(18 \%)\end{array}$ \\
\hline 11 & $\begin{array}{l}42330 . \\
69\end{array}$ & 236.2352317 & -0.0842 & 0.0017 & $\begin{array}{l}\text { H-4->LUMO } \quad(34 \%), \quad \text { H-4->L+1 } \\
(11 \%), H-3->\text { LUMO }(13 \%)\end{array}$ \\
\hline 12 & $\begin{array}{l}42561 . \\
36\end{array}$ & 234.9548724 & -6.7375 & 0.0035 & $\begin{array}{l}\text { H-4->LUMO }(11 \%), \quad \text { H-3->LUMO } \\
(12 \%)\end{array}$ \\
\hline 13 & $\begin{array}{l}42676 . \\
7\end{array}$ & 234.3198833 & -0.6225 & 0.0002 & $\mathrm{H}-5->\mathrm{L}+6(11 \%), \mathrm{H}-1->\mathrm{L}+8(20 \%)$ \\
\hline 14 & $\begin{array}{l}43036 . \\
43\end{array}$ & 232.3612891 & -6.4941 & 0.0024 & H-7->LUMO $(37 \%)$ \\
\hline 15 & $\begin{array}{l}43240 . \\
49\end{array}$ & 231.2647342 & 4.8238 & 0.0037 & $\begin{array}{l}\text { H-7->LUMO }(11 \%), \quad \text { H-6->LUMO } \\
(12 \%), \text { H-5->LUMO }(27 \%)\end{array}$ \\
\hline 16 & $\begin{array}{l}43331 . \\
63\end{array}$ & 230.7783051 & -5.8143 & 0.0035 & H-4->L+1 (11\%), H-2->L+1 (58\%) \\
\hline 17 & $\begin{array}{l}43445 . \\
35\end{array}$ & 230.1742071 & 0.738 & 0.0006 & $\begin{array}{lll}\text { H-6->LUMO } \quad(20 \%), & \text { H-6->L+1 } \\
(40 \%), H-5->L+1(24 \%) & \\
\end{array}$ \\
\hline 18 & 43490. & 229.9351582 & -1.5752 & 0.0014 & H-4->L+1 (21\%), H-3->L+1 (35\%), \\
\hline
\end{tabular}




\begin{tabular}{|c|c|c|c|c|c|}
\hline & 52 & & & & $\mathrm{H}-2->\mathrm{L}+1(21 \%)$ \\
\hline 19 & $\begin{array}{l}43749 . \\
43\end{array}$ & 228.5744195 & -1.2124 & 0.0015 & H-4->L+1 (50\%), H-3->L+1 (39\%) \\
\hline 20 & $\begin{array}{l}43935 . \\
74\end{array}$ & 227.6051193 & 2.5134 & 0.0039 & $\begin{array}{l}\text { H-10->LUMO (10\%), H-8->LUMO } \\
(62 \%)\end{array}$ \\
\hline 21 & $\begin{array}{l}44465 . \\
65\end{array}$ & 224.8926839 & 0.6641 & 0.0007 & $\begin{array}{lr}\text { H-6->LUMO } \quad(40 \%), & \text { H-6->L+1 } \\
(16 \%), \quad \text { H-5->LUMO } & (27 \%), \\
\text { H-5->L+1 }(13 \%) & \\
\end{array}$ \\
\hline 22 & $\begin{array}{l}44834 . \\
25\end{array}$ & 223.0437632 & -0.8019 & 0.0051 & $\begin{array}{ll}\text { H-10->L+1 } & (10 \%), H-8->L+1 \quad(10 \%), \\
H-1->L+2 & (20 \%), \quad \text { HOMO->L }+2 \\
(24 \%) & \end{array}$ \\
\hline 23 & $\begin{array}{l}44919 . \\
75\end{array}$ & 222.6192459 & 9.8036 & 0.0078 & $\begin{array}{lrr}\text { H-10->LUMO } \quad(23 \%), & \text { H-10->L }+1 \\
(10 \%), & \text { H-8->LUMO } & (10 \%), \\
\text { H-1->L+2 } & (13 \%), & \text { HOMO->L }+2 \\
(18 \%) & & \end{array}$ \\
\hline 24 & $\begin{array}{l}45121 . \\
39\end{array}$ & 221.6243974 & -16.3105 & 0.0019 & $\begin{array}{l}\text { H-1->L+2 } \quad(16 \%), \quad \text { HOMO->L+2 } \\
(21 \%), \text { HOMO->L+3 }(47 \%)\end{array}$ \\
\hline 25 & $\begin{array}{l}45173 . \\
81\end{array}$ & 221.3671915 & -2.0194 & 0.0032 & $\mathrm{H}-8->\mathrm{L}+1(25 \%), \mathrm{H}-7->\mathrm{L}+1(52 \%)$ \\
\hline 26 & $\begin{array}{l}45198 . \\
82\end{array}$ & 221.2447343 & 0.7726 & 0.0024 & $\begin{array}{lll}\text { H-10->LUMO } \quad(28 \%), & \text { H- } 8->L+1 \\
(22 \%), H-7->L+1(31 \%) & \\
\end{array}$ \\
\hline 27 & $\begin{array}{l}45398 . \\
84\end{array}$ & 220.269932 & 22.302 & 0.0242 & $\begin{array}{l}\text { H-1->L+3 (19\%), H-1->L+6 (11\%), } \\
\text { HOMO->L+5 }(10 \%)\end{array}$ \\
\hline 28 & $\begin{array}{l}45510 . \\
95\end{array}$ & 219.7273183 & -5.4575 & 0.0022 & $\begin{array}{l}\text { H-1->L+4 }(37 \%), \text { H-1->L+5 (11\%), } \\
\text { HOMO->L+4 }(31 \%)\end{array}$ \\
\hline 29 & $\begin{array}{l}45768 . \\
25\end{array}$ & 218.4920903 & -33.6701 & 0.0067 & $\begin{array}{l}\mathrm{H}-1->\mathrm{L}+3 \quad(24 \%), \quad \text { HOMO->L+4 } \\
(13 \%), \mathrm{HOMO}->\mathrm{L}+5(25 \%)\end{array}$ \\
\hline 30 & $\begin{array}{l}45845 . \\
68\end{array}$ & 218.1230743 & -7.6272 & 0.0041 & $\mathrm{H}-10->\mathrm{L}+1(39 \%), \mathrm{H}-8->\mathrm{L}+1(28 \%)$ \\
\hline
\end{tabular}


Table S5. Cartesian coordinate of the dominant conformer of 1

\section{Standard orientation:}

\begin{tabular}{|c|c|c|c|c|c|}
\hline \multirow{2}{*}{$\begin{array}{l}\text { Center } \\
\text { Number }\end{array}$} & \multirow{2}{*}{$\begin{array}{l}\text { Atomic } \\
\text { Number }\end{array}$} & \multirow{2}{*}{$\begin{array}{l}\text { Atomic } \\
\text { Type }\end{array}$} & \multicolumn{3}{|c|}{ Coordinates (Angstroms) } \\
\hline & & & $\mathbf{X}$ & $\mathbf{Y}$ & $\mathbf{Z}$ \\
\hline 1 & 6 & 0 & -0.18733 & -0.9084 & -1.25394 \\
\hline 2 & 6 & 0 & -1.74835 & -0.94868 & -1.18334 \\
\hline 3 & 6 & 0 & -2.28882 & 0.37235 & -1.77248 \\
\hline 4 & 6 & 0 & -1.64163 & 1.598708 & -1.12331 \\
\hline 5 & 8 & 0 & -0.22106 & 1.529855 & -1.26906 \\
\hline 6 & 6 & 0 & 0.369908 & 0.396912 & -0.63198 \\
\hline 7 & 6 & 0 & -2.10209 & 2.906913 & -1.76448 \\
\hline 8 & 6 & 0 & -1.52541 & 4.181495 & -1.10977 \\
\hline 9 & 6 & 0 & -1.99128 & 4.409078 & 0.313625 \\
\hline 10 & 6 & 0 & -2.27972 & -2.18326 & -1.95084 \\
\hline 11 & 6 & 0 & -3.8142 & -2.31881 & -2.09876 \\
\hline 12 & 6 & 0 & -4.58748 & -2.51707 & -0.8067 \\
\hline 13 & 6 & 0 & 1.882835 & 0.53049 & -0.8234 \\
\hline 14 & 6 & 0 & 2.480113 & 1.760109 & -0.11169 \\
\hline 15 & 6 & 0 & 3.967627 & 1.914726 & -0.35064 \\
\hline 16 & 6 & 0 & 0.463034 & -2.18756 & -0.70163 \\
\hline 17 & 6 & 0 & 0.534775 & -2.40433 & 0.805218 \\
\hline 18 & 6 & 0 & 1.412687 & -3.59425 & 1.236665 \\
\hline 19 & 6 & 0 & 2.899874 & -3.29617 & 1.244926 \\
\hline 20 & 6 & 0 & 4.455054 & 2.863359 & -1.25877 \\
\hline 21 & 6 & 0 & 5.824308 & 2.991476 & -1.49994 \\
\hline 22 & 6 & 0 & 6.731984 & 2.167525 & -0.83342 \\
\hline 23 & 6 & 0 & 6.259226 & 1.21654 & 0.073638 \\
\hline 24 & 6 & 0 & 4.89063 & 1.092847 & 0.312186 \\
\hline 25 & 6 & 0 & 3.688424 & -3.4765 & 0.098591 \\
\hline 26 & 6 & 0 & 5.054351 & -3.18956 & 0.119049 \\
\hline 27 & 6 & 0 & 5.658922 & -2.71825 & 1.286103 \\
\hline 28 & 6 & 0 & 4.885491 & -2.53234 & 2.43382 \\
\hline 29 & 6 & 0 & 3.519403 & -2.81959 & 2.40935 \\
\hline 30 & 6 & 0 & -4.42116 & -3.68453 & -0.04318 \\
\hline 31 & 6 & 0 & -5.13105 & -3.87553 & 1.140662 \\
\hline 32 & 6 & 0 & -6.02541 & -2.90011 & 1.590852 \\
\hline 33 & 6 & 0 & -6.20561 & -1.73706 & 0.843798 \\
\hline 34 & 6 & 0 & -5.49376 & -1.55067 & -0.34537 \\
\hline 35 & 6 & 0 & -1.20032 & 4.027161 & 1.405863 \\
\hline 36 & 6 & 0 & -1.64284 & 4.219048 & 2.716236 \\
\hline 37 & 6 & 0 & -2.8885 & 4.799752 & 2.957803 \\
\hline 38 & 6 & 0 & -3.68673 & 5.187882 & 1.879518 \\
\hline 39 & 6 & 0 & -3.23975 & 4.993511 & 0.572467 \\
\hline
\end{tabular}




\begin{tabular}{|c|c|c|c|c|c|}
\hline 40 & 8 & 0 & -2.08011 & -1.02379 & 0.211983 \\
\hline 41 & 1 & 0 & 0.077213 & -0.89585 & -2.31754 \\
\hline 42 & 8 & 0 & 0.869176 & -3.03199 & -1.48479 \\
\hline 43 & 1 & 0 & -3.37493 & 0.419713 & -1.62388 \\
\hline 44 & 1 & 0 & -2.10539 & 0.396382 & -2.85518 \\
\hline 45 & 1 & 0 & -1.89055 & 1.600618 & -0.05169 \\
\hline 46 & 1 & 0 & 0.118301 & 0.424219 & 0.439496 \\
\hline 47 & 1 & 0 & -3.19926 & 2.9415 & -1.7296 \\
\hline 48 & 1 & 0 & -1.814 & 2.891892 & -2.82402 \\
\hline 49 & 1 & 0 & -1.81549 & 5.040644 & -1.72805 \\
\hline 50 & 1 & 0 & -0.4327 & 4.123563 & -1.14033 \\
\hline 51 & 1 & 0 & -1.88928 & -3.08389 & -1.46392 \\
\hline 52 & 1 & 0 & -1.84566 & -2.17323 & -2.95834 \\
\hline 53 & 1 & 0 & -3.99399 & -3.18407 & -2.75054 \\
\hline 54 & 1 & 0 & -4.21896 & -1.45287 & -2.63483 \\
\hline 55 & 1 & 0 & 2.088555 & 0.592271 & -1.89961 \\
\hline 56 & 1 & 0 & 2.383122 & -0.3764 & -0.46162 \\
\hline 57 & 1 & 0 & 2.285673 & 1.672259 & 0.966876 \\
\hline 58 & 1 & 0 & 1.953762 & 2.655837 & -0.45691 \\
\hline 59 & 1 & 0 & -0.50478 & -2.54426 & 1.130035 \\
\hline 60 & 1 & 0 & 0.858084 & -1.48166 & 1.301758 \\
\hline 61 & 1 & 0 & 1.204071 & -4.43875 & 0.572304 \\
\hline 62 & 1 & 0 & 1.105153 & -3.89214 & 2.246128 \\
\hline 63 & 1 & 0 & 3.752953 & 3.510645 & -1.78034 \\
\hline 64 & 1 & 0 & 6.180781 & 3.736988 & -2.20644 \\
\hline 65 & 1 & 0 & 7.798669 & 2.266245 & -1.01748 \\
\hline 66 & 1 & 0 & 6.956576 & 0.568344 & 0.598277 \\
\hline 67 & 1 & 0 & 4.536259 & 0.346246 & 1.019637 \\
\hline 68 & 1 & 0 & 3.220132 & -3.83956 & -0.81125 \\
\hline 69 & 1 & 0 & 5.648307 & -3.33829 & -0.77909 \\
\hline 70 & 1 & 0 & 6.724451 & -2.50406 & 1.302976 \\
\hline 71 & 1 & 0 & 5.346196 & -2.17389 & 3.351112 \\
\hline 72 & 1 & 0 & 2.925826 & -2.68323 & 3.31153 \\
\hline 73 & 1 & 0 & -3.73096 & -4.45249 & -0.38516 \\
\hline 74 & 1 & 0 & -4.98842 & -4.78831 & 1.713116 \\
\hline 75 & 1 & 0 & -6.57785 & -3.04907 & 2.51445 \\
\hline 76 & 1 & 0 & -6.90269 & -0.97387 & 1.179433 \\
\hline 77 & 1 & 0 & -5.65417 & -0.64714 & -0.93002 \\
\hline 78 & 1 & 0 & -0.22644 & 3.578603 & 1.224043 \\
\hline 79 & 1 & 0 & -1.01116 & 3.918142 & 3.548227 \\
\hline 80 & 1 & 0 & -3.23281 & 4.953853 & 3.977058 \\
\hline 81 & 1 & 0 & -4.65541 & 5.648647 & 2.056638 \\
\hline 82 & 1 & 0 & -3.86454 & 5.307569 & -0.26177 \\
\hline
\end{tabular}


Table S6. Cartesian coordinate of the dominant conformer of 2

\begin{tabular}{|c|c|c|c|c|c|}
\hline \multicolumn{6}{|c|}{ Standard orientation: } \\
\hline \multirow{2}{*}{$\begin{array}{l}\text { Center } \\
\text { Number }\end{array}$} & \multirow{2}{*}{$\begin{array}{l}\text { Atomic } \\
\text { Number }\end{array}$} & \multirow{2}{*}{$\begin{array}{l}\text { Atomic } \\
\text { Type }\end{array}$} & \multicolumn{3}{|c|}{ Coordinates (Angstroms) } \\
\hline & & & $\mathbf{X}$ & $\mathbf{Y}$ & $\mathbf{Z}$ \\
\hline 1 & 6 & 0 & 6.276997 & 2.254955 & 0.286723 \\
\hline 2 & 6 & 0 & 5.862554 & 3.253728 & -0.59926 \\
\hline 3 & 6 & 0 & 4.558258 & 3.74921 & -0.51463 \\
\hline 4 & 6 & 0 & 3.673664 & 3.249486 & 0.445538 \\
\hline 5 & 6 & 0 & 4.075573 & 2.244088 & 1.338659 \\
\hline 6 & 6 & 0 & 5.389759 & 1.759077 & 1.245943 \\
\hline 7 & 6 & 0 & 3.120025 & 1.677462 & 2.372379 \\
\hline 8 & 6 & 0 & 2.320895 & 0.459082 & 1.872875 \\
\hline 9 & 6 & 0 & 1.116128 & 0.784965 & 0.995751 \\
\hline 10 & 6 & 0 & 0.47843 & -0.37537 & 0.242135 \\
\hline 11 & 6 & 0 & 0.59431 & -0.30975 & -1.30545 \\
\hline 12 & 8 & 0 & 0.644504 & 1.911223 & 0.949799 \\
\hline 13 & 6 & 0 & 2.920696 & -2.53577 & -1.1806 \\
\hline 14 & 6 & 0 & 4.038545 & -2.4338 & -0.33626 \\
\hline 15 & 6 & 0 & 4.262341 & -3.36734 & 0.679029 \\
\hline 16 & 6 & 0 & 3.369847 & -4.42771 & 0.867207 \\
\hline 17 & 6 & 0 & 2.256716 & -4.54456 & 0.030724 \\
\hline 18 & 6 & 0 & 2.036788 & -3.60781 & -0.98424 \\
\hline 19 & 6 & 0 & 2.039614 & -0.18158 & -1.83666 \\
\hline 20 & 6 & 0 & 2.685156 & -1.51655 & -2.27821 \\
\hline 21 & 8 & 0 & -0.13947 & 0.784002 & -1.87155 \\
\hline 22 & 6 & 0 & -1.87608 & 3.217757 & -1.43994 \\
\hline 23 & 6 & 0 & -2.04673 & 2.054057 & -2.44194 \\
\hline 24 & 6 & 0 & -4.25871 & 3.618735 & -0.663 \\
\hline 25 & 6 & 0 & -5.24918 & 3.648087 & 0.321073 \\
\hline 26 & 6 & 0 & -4.93521 & 3.307102 & 1.641239 \\
\hline 27 & 6 & 0 & -3.62579 & 2.941594 & 1.961772 \\
\hline 28 & 6 & 0 & -2.63637 & 2.912093 & 0.972412 \\
\hline 29 & 6 & 0 & -2.9383 & 3.247804 & -0.35565 \\
\hline 30 & 8 & 0 & -1.76261 & -2.73024 & -2.11048 \\
\hline 31 & 6 & 0 & -1.5626 & 0.689893 & -1.92978 \\
\hline 32 & 6 & 0 & -2.02717 & -0.46598 & -2.87949 \\
\hline 33 & 6 & 0 & -2.47095 & -1.73623 & -2.16572 \\
\hline 34 & 6 & 0 & -3.87132 & -1.71372 & -1.5615 \\
\hline
\end{tabular}




\begin{tabular}{|c|c|c|c|c|c|}
\hline 35 & 6 & 0 & -4.17205 & -2.84409 & -0.56053 \\
\hline 36 & 6 & 0 & -2.36526 & -3.38937 & 1.147286 \\
\hline 37 & 6 & 0 & -3.51275 & -2.66426 & 0.794449 \\
\hline 38 & 6 & 0 & -4.04648 & -1.76541 & 1.731899 \\
\hline 39 & 6 & 0 & -3.45164 & -1.59284 & 2.983914 \\
\hline 40 & 6 & 0 & -2.30959 & -2.32441 & 3.324134 \\
\hline 41 & 6 & 0 & -1.76857 & -3.22306 & 2.400798 \\
\hline 42 & 1 & 0 & 7.29235 & 1.868311 & 0.237664 \\
\hline 43 & 1 & 0 & 6.55106 & 3.645732 & -1.3438 \\
\hline 44 & 1 & 0 & 4.227094 & 4.529521 & -1.19584 \\
\hline 45 & 1 & 0 & 2.65907 & 3.633791 & 0.502527 \\
\hline 46 & 1 & 0 & 5.725318 & 0.990067 & 1.940205 \\
\hline 47 & 1 & 0 & 3.692228 & 1.372032 & 3.25692 \\
\hline 48 & 1 & 0 & 2.414543 & 2.45055 & 2.694715 \\
\hline 49 & 1 & 0 & 2.967275 & -0.25826 & 1.35196 \\
\hline 50 & 1 & 0 & 1.909915 & -0.09908 & 2.731069 \\
\hline 51 & 1 & 0 & 0.921013 & -1.32339 & 0.56553 \\
\hline 52 & 1 & 0 & -0.5822 & -0.40717 & 0.519411 \\
\hline 53 & 1 & 0 & 0.170375 & -1.24941 & -1.68089 \\
\hline 54 & 1 & 0 & 4.746514 & -1.6198 & -0.48352 \\
\hline 55 & 1 & 0 & 5.138929 & -3.27343 & 1.315951 \\
\hline 56 & 1 & 0 & 3.546393 & -5.15968 & 1.651452 \\
\hline 57 & 1 & 0 & 1.561394 & -5.37057 & 0.160413 \\
\hline 58 & 1 & 0 & 1.16765 & -3.7112 & -1.63057 \\
\hline 59 & 1 & 0 & 1.993916 & 0.473016 & -2.71394 \\
\hline 60 & 1 & 0 & 2.687454 & 0.326184 & -1.11276 \\
\hline 61 & 1 & 0 & 2.055895 & -1.96599 & -3.05742 \\
\hline 62 & 1 & 0 & 3.64532 & -1.27731 & -2.75379 \\
\hline 63 & 1 & 0 & -1.92082 & 4.158387 & -2.00558 \\
\hline 64 & 1 & 0 & -0.88025 & 3.160589 & -0.99034 \\
\hline 65 & 1 & 0 & -3.105 & 1.972479 & -2.72061 \\
\hline 66 & 1 & 0 & -1.48766 & 2.272282 & -3.3618 \\
\hline 67 & 1 & 0 & -4.51173 & 3.90072 & -1.68426 \\
\hline 68 & 1 & 0 & -6.26234 & 3.946261 & 0.060845 \\
\hline 69 & 1 & 0 & -5.70244 & 3.335308 & 2.411323 \\
\hline 70 & 1 & 0 & -3.36768 & 2.683341 & 2.986358 \\
\hline 71 & 1 & 0 & -1.61718 & 2.634278 & 1.22725 \\
\hline 72 & 1 & 0 & -1.96642 & 0.522904 & -0.91936 \\
\hline 73 & 1 & 0 & -2.87442 & -0.10457 & -3.47597 \\
\hline 74 & 1 & 0 & -1.2126 & -0.70959 & -3.56788 \\
\hline 75 & 1 & 0 & -4.05734 & -0.72763 & -1.11462 \\
\hline 76 & 1 & 0 & -4.56511 & -1.78021 & -2.41562 \\
\hline 77 & 1 & 0 & -5.26011 & -2.89315 & -0.42651 \\
\hline
\end{tabular}




\begin{tabular}{|r|r|r|r|r|r|}
\hline 78 & 1 & 0 & -3.85958 & -3.79615 & -1.00175 \\
\hline 79 & 1 & 0 & -1.9348 & -4.08217 & 0.429172 \\
\hline 80 & 1 & 0 & -4.9406 & -1.19626 & 1.482841 \\
\hline 81 & 1 & 0 & -3.88427 & -0.89307 & 3.694777 \\
\hline 82 & 1 & 0 & -1.84877 & -2.1972 & 4.300696 \\
\hline 83 & 1 & 0 & -0.8812 & -3.79798 & 2.654943 \\
\hline
\end{tabular}

